\title{
Myosin-1a Is Critical for Normal Brush Border Structure and Composition
}

\author{
Matthew J. Tyska, ${ }^{*+}$ Andrew T. Mackey, ${ }^{*}$ Jian-Dong Huang, ${ }^{\ddagger}$ Neil G. Copeland, ${ }^{\ddagger}$ \\ Nancy A. Jenkins, ${ }^{\ddagger}$ and Mark S. Mooseker*|II
}

\author{
*Department of Molecular, Cellular, and Developmental Biology, Yale University, New Haven, CT 06520; \\ ‡National Cancer Institute, National Institutes of Health, Frederick, MD 21702; and Departments of "Cell \\ Biology and IIPathology, Yale University, New Haven, CT 06520
}

Submitted December 23, 2004; Revised February 14, 2005; Accepted February 28, 2005

Monitoring Editor: Paul Matsudaira

\begin{abstract}
To develop our understanding of myosin-1a function in vivo, we have created a mouse line null for the myosin-1a gene. Myosin-1a knockout mice demonstrate no overt phenotypes at the whole animal level but exhibit significant perturbations and signs of stress at the cellular level. Among these are defects in microvillar membrane morphology, distinct changes in brush-border organization, loss of numerous cytoskeletal and membrane components from the brush border, and redistribution of intermediate filament proteins into the brush border. We also observed significant ectopic recruitment of another short-tailed class I motor, myosin-1c, into the brush border of knockout enterocytes. This latter finding, a clear demonstration of functional redundancy among vertebrate myosins-I, may account for the lack of a whole animal phenotype. Nevertheless, these results indicate that myosin-1a is a critical multifunctional component of the enterocyte, required for maintaining the normal composition and highly ordered structure of the brush border.
\end{abstract}

\section{INTRODUCTION}

The brush border (BB) domain found at the apical surface of the enterocyte is one of the most exquisitely well ordered cytoskeletal structures observed in nature and has served as a powerful model system for investigating membrane-cytoskeleton interactions for more than three decades. Consisting of tightly packed arrays of microvilli (MV) that are nearly identical in length and diameter (Mooseker, 1985), the $\mathrm{BB}$ serves as the primary site for nutrient absorption as well as the main barrier to intestinal flora and pathogens introduced to the gastrointestinal tract. Within a single MV, the plasma membrane is linked to a supporting polarized actin bundle with a spiraling array of bridges composed of the actin-based motor protein, myosin-1a (Myola, previously known as BB myosin I; Mukherjee and Staehelin, 1971; Mooseker and Tilney, 1975; Matsudaira and Burgess, 1979). Myola is one of eight vertebrate class I myosins (Berg et al., 2001); monomeric motors with the capacity for binding multiple calmodulin (CaM) light chains in a $\mathrm{Ca}^{2+}$ sensitive manner and the ability to interact directly with membranes

This article was published online ahead of print in $M B C$ in Press (http:/ / www.molbiolcell.org/cgi/doi/10.1091/mbc.E04-12-1116) on March 9, 2005.

Present addresses: ${ }^{+}$Department of Cell and Developmental Biology, Vanderbilt University, Nashville, TN 37232; \$Department of Biochemistry, University of Hong Kong, Hong Kong, China.

Address correspondence to: Matthew J. Tyska (matthew.tyska@ vanderbilt.edu).

Abbreviations used: AP, alkaline phosphatese; $\mathrm{BB}$, brush border; DRM, detergent-resistant membrane; Gal4, galectin-4; MV, microvillus/i/ar; Myo, myosin; SI, sucrase isomaltase; TEM, transmission electron microscopy; SEM, scanning electron microscopy. by virtue of highly basic regions (TH1) in their C-terminal tail domains (Mooseker and Cheney, 1995).

The biochemical and biophysical properties of class I myosins, and specifically purified Myo1a, have been characterized extensively. Myola is a slow, plus-end-directed motor (Mooseker and Cheney, 1995) that demonstrates a low duty ratio (5-10\%) (Jontes et al., 1997) and a two-phase unitary displacement that may be indicative of load-dependent stepping kinetics (Veigel et al., 1999). Studies on the TH1 domain of Myola have revealed that it binds directly to acidic phospholipids with a moderate affinity (Mooseker and Cheney, 1995). More recently, fluorescence recovery after photobleaching studies have shown that TH1 plays an important role in the steady-state localization of Myola at the membrane and may govern the dynamics of this motor within the BB (Tyska and Mooseker, 2002a). Together, these studies establish the potential for Myo1a to function as a membraneassociated motor; e.g., powering the directed movement of subcellular membranes or developing tension in the plasma membrane.

Given its striking localization in the MV, Myola is uniquely situated to participate in a variety of processes critical for normal BB structure and function. Proposed functional hypotheses include 1) mechanochemical gating of solute or ion transporters in the MV membrane; 2) $\mathrm{Ca}^{2+}$ buffering within the $\mathrm{MV}$, via the high $\mathrm{Ca}^{2+}$ binding capacity of bound CaM light chains; 3) mechanical stabilization of MV structure, perhaps through the development of tension in the MV membrane (Mooseker and Cheney, 1995); 4) apical transport of vesicular cargo derived from the Golgi (Fath et al., 1994); and 5) regulation of the endosomal and lysosomal compartments (Durrbach et al., 1996; Durrbach et al., 2000). More recently, our laboratory has shown that Myola is a component of lipid rafts in the MV membrane and is required for the retention of sucrase-isomaltase (SI) in the BB (Tyska and Mooseker, 2004). However, this and all previous 
A.
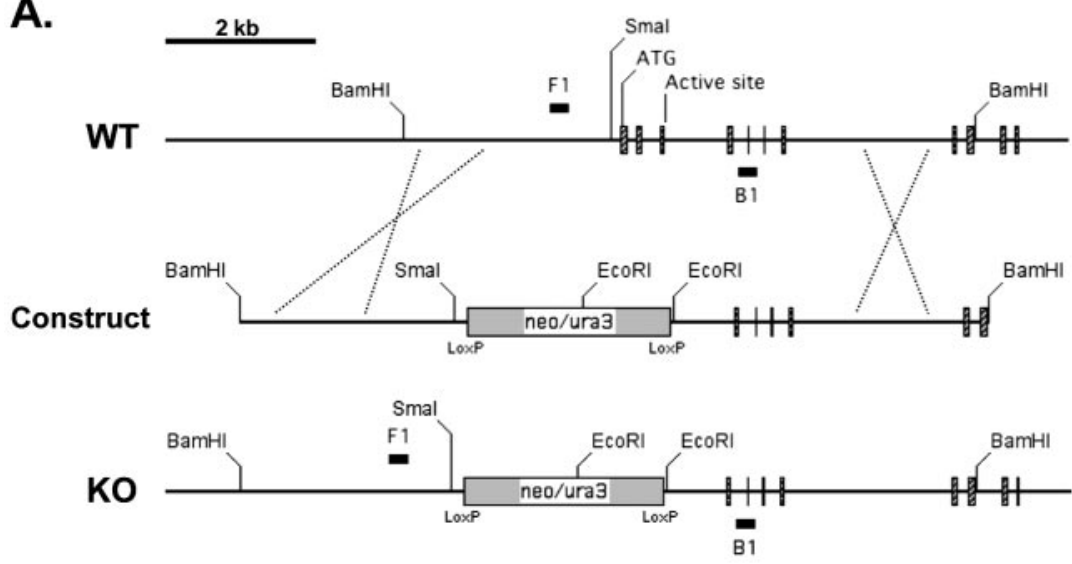

B.

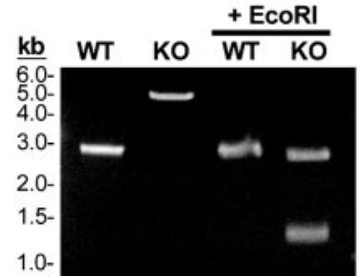

D.

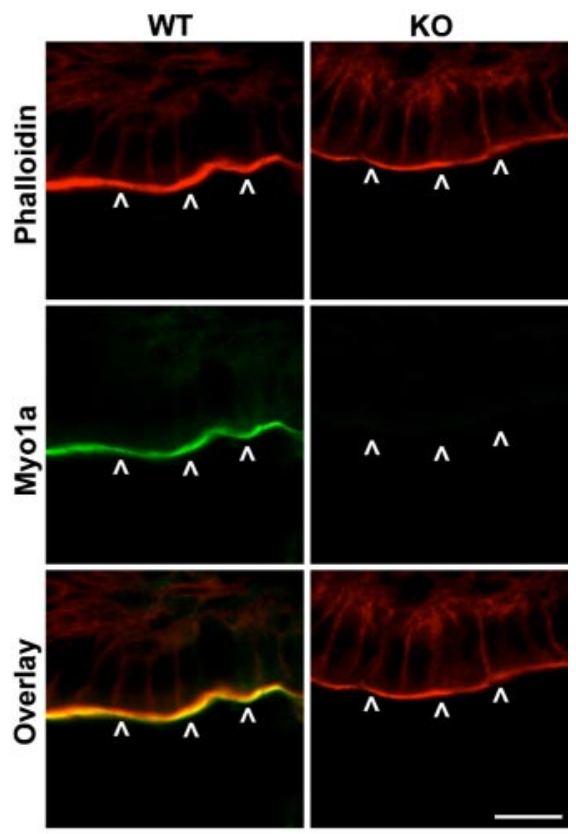

Figure 1. (A) Schematic representation of Myo1a genomic structure (WT), structure of the construct devised for Myo1a targeting (construct), and the result following recombination (KO). The neo/ura selection cassette is positioned to replace the first three exons of Myola. Exons are shown as cross-hatched boxes; native restriction sites (SmaI and BamHI), ATG start site, and ATP binding sites are flagged for reference. F1 and B1 mark the positions of genomic PCR primer binding sites. (B) PCR with genomic template from WT and homozygous KO mice. Products of expected size were observed (2808 base pairs for WT and 5119 base pairs for KO); cutting with EcoRI confirmed the introduction of two new restriction sites in the KO. Note that three KO fragments are produced with EcoRI (2623, 1303, and 1193 base pairs), but two are nearly identical in size and not resolved by the gel. (C) Anti-Myola immunoblots on whole gut (WG), mucosal homogenate (MH), and crude BB (CBB) fractions from WT and homozygous KO small intestines (loaded for equal total protein). (D) Staining of WT and KO duodenal sections with anti-Myola (green) and phalloidin (red). Bar, $10 \mu \mathrm{m}$.

studies have been restricted to the use of in vitro model systems (e.g., assays with purified proteins or cultured cells).

In an effort to develop our understanding of Myola function in vivo, we have created a mouse line null for the Myola gene. Although Myo1a knockout (KO) mice demonstrate no overt phenotypes at the whole animal level, our multifaceted phenotypic characterization has revealed a number of significant perturbations at the cellular level. Among these are defects in MV membrane morphology, distinct changes in BB organization, loss of numerous cytoskeletal and membrane components from the $\mathrm{BB}$, and redistribution of intermediate filament proteins into the $\mathrm{BB}$. In addition, we observed significant ectopic recruitment of another short-tailed class I motor, myosin-1c (Myo1c), into the BB of KO enterocytes. This represents the first clear demonstration of functional redundancy among vertebrate myosins- 1 and also may account for the limited severity of the observed phenotypes. Nevertheless, the unexpected diversity of the effects induced by eliminating Myola expression indicates that this motor is a critical multifunctional component of the BB. These studies also provide us with an in vivo framework for the interpretation of previous in vitro studies, and a foundation for the development of new functional hypotheses regarding this class I myosin.

\section{MATERIALS AND METHODS}

\section{Generation of the Myo1a Null Mutation}

Mouse genomic DNA containing the Myo1a gene was obtained by screening a mouse $129 \mathrm{SV}$ genomic library. An $\sim 9-\mathrm{kb}$ Bam $\mathrm{HI}$ restriction fragment was cloned into a yeast shuttle vector containing the TK gene to obtain pTK Myo1a. DNA primers with 50 base pairs of Myo1a sequence at the ends were synthesized and used to amplify a neo/URA3 fragment. The PCR product was purified and cotransformed with pTK Myo1a into yeast strain H1515. Yeast clones positive for URA3 were selected. The neo/URA3 fragment replaced the first three Myo1a exons via yeast-mediated homologous recombination. Embryonic stem (ES) cell transfection and selection were performed as described previously (Wilson et al., 2000). Proper positioning of the KO cassette was confirmed by genomic PCR (Expand; Roche Diagnostics, Indianapolis, IN) by using primers that flanked the first four exons of Myo1a (Figure 1A, F1 and B1) and southern blots with probes positioned 5' and 3' to the targeted region. After germline transmission of the $\mathrm{KO}$ allele, the resulting heterozygotes were bred to generate animals homozygous for the Myola deletion. Although our initial phenotypic studies were performed on mice with a mixed genetic background, $\mathrm{KO}$ mice were eventually backcrossed to a pure $129 \mathrm{X} 1 / \mathrm{SvJ}$ background, enabling us to use commercially available 129X1/SvJ mice as wild-type (WT) controls. All procedures involving mice were performed in accordance with Yale-IACUC protocol \#10214.

\section{Histology}

For histological staining with hematoxylin and eosin (H\&E), paraffin sections were prepared from the small intestine. Briefly, duodenal segments were excised from WT and KO mice, washed with room temperature (RT) phosphate-buffered saline, and fixed overnight in $4 \%$ paraformaldehyde (PFA)/ phosphate-buffered saline. Tissue fragments were then embedded in paraffin, sectioned, and stained by Research Histology at Yale University School of Medicine. For TUNEL assays (Roche), unstained sections were deparaffinized in xylene, rehydrated in a graded ethanol series, and stained using protocols provided by the manufacturer. For the quantification of terminal deoxynucleotidyl transferase dUTP nick-end labeling (TUNEL) intensities in MetaMorph (version 5.0; Universal Imaging, Downingtown, PA), confocal images of TUNEL signal from whole sections were thresholded to eliminate background and then binarized so that all TUNEL-positive pixels were equivalent to 1 . The total integrated intensity from each section (proportional to the number of TUNEL-positive cells) was then measured and normalized to the highest WT value for plotting in SigmaPlot (version 7.0; Systat Software, Point Richmond, CA). 
A.

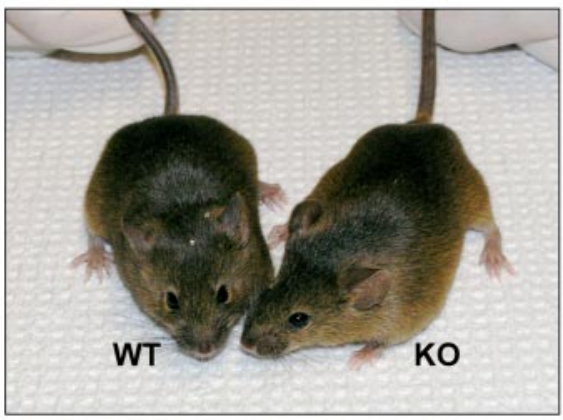

B.

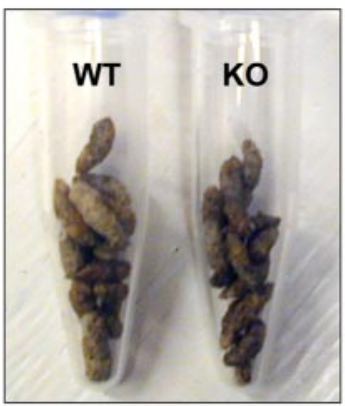

D.

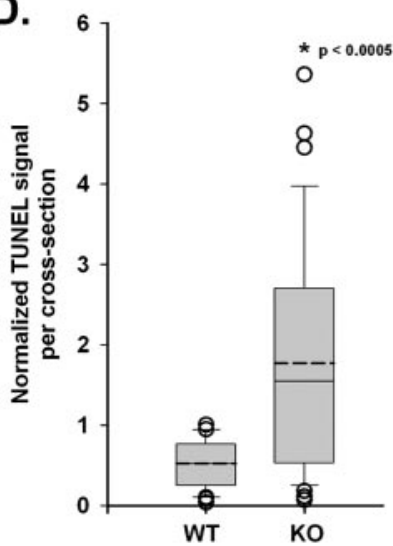

E.

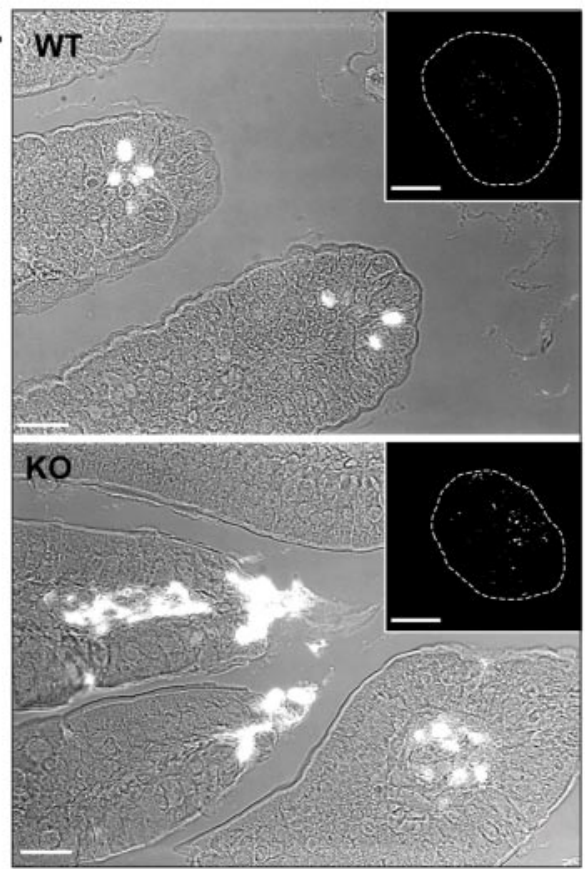

C.
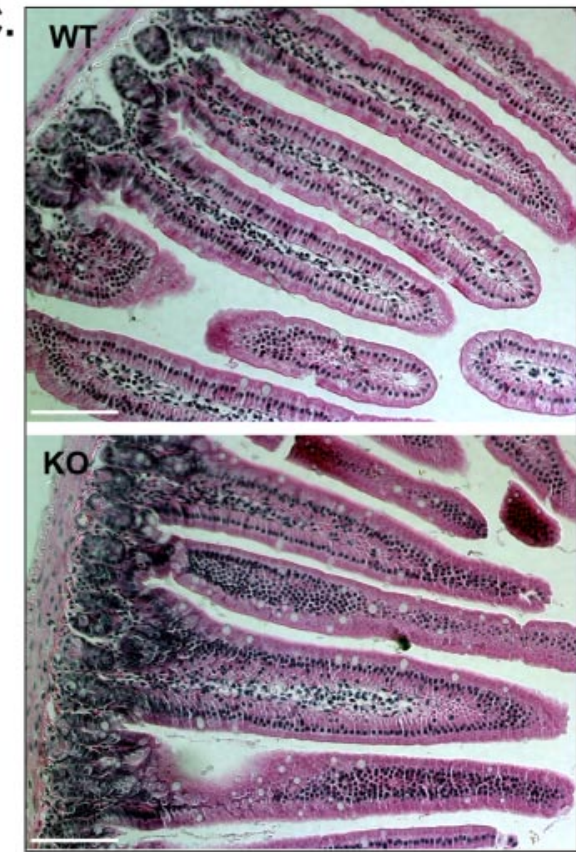

F.

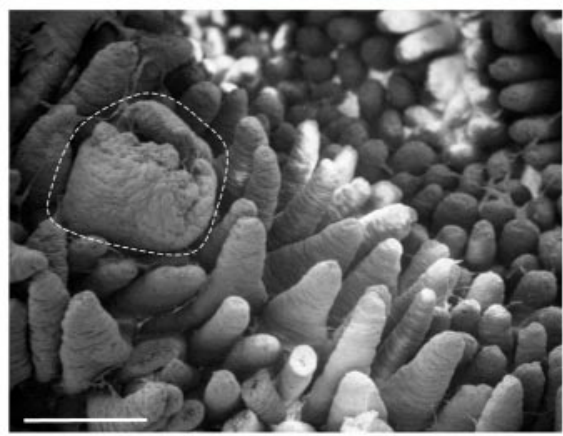

Figure 2. (A) Age-matched $\mathrm{WT}$ and $\mathrm{KO}$ mice ( $8 \mathrm{wk}$ old) appear no different. (B) Total stool produced over a 6-h period from five WT and five KO mice. (C) Paraffin sections of WT and KO duodenum stained with H\&E. Bars, $100 \mu \mathrm{m}$. (D) KO paraffin sections exhibit significantly higher levels of TUNEL staining (WT, $0.5 \pm 0.3, \mathrm{n}=27$ sections vs. KO, $1.8 \pm 1.5, \mathrm{n}=31$ sections; ${ }^{*} \mathrm{p}<0.0005$ ). Values for the total TUNEL intensity per section were normalized to the highest WT value (see Materials and Methods). WT and KO data are presented as box-plots; solid line within the box corresponds to the median; dashed line corresponds to the mean; edges of the box are the 25th and 75th percentiles; whiskers are the 10th and 90th percentiles; and open circles represent outliers. (E) Combined differential interference contrast and fluorescent images of villus tips show apoptotic cells (bright white regions) in WT and KO sections. Bars, $10 \mu \mathrm{m}$. Insets, TUNEL signal from the entire cross section (outlined with white dashed lines). Bars, $500 \mu \mathrm{m}$. (F) SEM of an enlarged and damaged villus in KO duodenum (see white dashed line). Bar, $500 \mu \mathrm{m}$.

\section{Immunocytochemistry}

Immunostaining was performed as described previously (Heintzelman and Mooseker, 1990b). The following dilutions were used for primary antibodies: anti-Myo1a (1:250), anti-SI (1:2000), anti-alkaline phosphatase (AP) (1:2000), antiMyo6 (10 $\mu \mathrm{g} / \mathrm{ml})$, anti-wide-spectrum cytokeratin (WSCK) (1:200), and antiMyo1c $(10 \mu \mathrm{g} / \mathrm{ml})$. Secondary antibodies and phalloidin conjugated to Alexa-488 or Alexa-568 (Molecular Probes, Eugene, OR) were used at 1:500.

\section{Cell Fractionation, BB Isolation, and Detergent-resistant Membrane (DRM) Microdomain Preparation}

Cell fractionation and BB isolation were performed as described previously (Mooseker and Tilney, 1975). For the membrane shedding assay (Figure 8), phalloidin stabilized BBs in $\mathrm{A}^{\prime}$ (1 mM EGTA, $75 \mathrm{mM} \mathrm{KCl}, 5 \mathrm{mM} \mathrm{MgCl} \mathrm{mM}_{2}, 1 \mathrm{mM}$ dithiothreitol, $1 \mathrm{mM}$ Pefabloc, and $10 \mathrm{mM}$ imidazole, $\mathrm{pH}$ 7.2) were exposed to $1 \mathrm{mM}$ MgATP for $15 \mathrm{~min}$ at RT. Samples were then fixed with $4 \%$ PFA and labeled with concanavalin A (ConA)-Alexa-488 (Molecular Probes) as described previously (Tyska and Mooseker, 2004). For the fractionation shown in Figure 10, whole cell lysates were spun at $1500 \times g$ for $10 \mathrm{~min}$ to generate low-speed samples (low-speed pellet [LSP] and low-speed supernatant [LSS]) The LSS was then spun at $20,000 \times g$ for $10 \mathrm{~min}$ to generate high-speed samples (high-speed pellet [HSP] and high-speed supernatant [HSS]), and the HSS was spun at $100,000 \times g$ for 60 min to create ultrahigh-speed samples (ultraspeed pellet [USP] and ultraspeed supernatant). All pellet fractions were resuspended in volumes equal to the initial lysate and equal volumes of all fractions were subject to SDS-PAGE as described below. Preparation of DRMs and subsequent Optiprep density gradient analysis was performed as described previously (Tyska and Mooseker, 2004). However, for the work presented here, DRMs were derived from purified intact BBs and not MV.

\section{Light Microscopy}

All of the images presented here were acquired on one of three microscopes: 1) Zeiss Axiovert with $4 \times / 0.1$ and $63 \times / 1.4$ PlanApo objectives coupled to a laser scanning confocal unit (MRC-1024; Bio-Rad, Hercules, CA) (Figures 1D 2E, inset; 8A, D-G, H, K-N; 9D; and 10B); 2) Nikon Diaphot 300 with $20 \times / 0.75$ and $40 \times / 1.2$ PlanApo objectives coupled to a Photometrics CoolSNAP HQ cooled charge-coupled device camera (Figures 2E and 8, B and I) and 3) Nikon Eclipse E800 with a $20 \times / 0.75$ PlanApo objective coupled to SPOT imaging hardware and software (SPOT Image, Chantilly, VI; Figure 2C). Images were LUT stretched using MetaMorph (version 5.0) and assembled into figures by using PowerPoint (version X; Microsoft, Redmond, WA) All images comparing WT and KO preparations were acquired with identical 
parameters (e.g., pinhole diameter, detector gain) and postprocessed in an identical manner.

\section{Electron Microscopy}

Segments of WT and KO intestine were fixed and dehydrated for transmission electron microscopy (TEM) and scanning electron microscopy (SEM) as described previously (Heintzelman and Mooseker, 1990b). Isolated BBs were processed according to established methods (Mooseker and Tilney, 1975) TEM negatives were digitized at $1200 \mathrm{dpi}$, and morphometry (measurements of angles, lengths, and diameters as in Figure 7) was performed using MetaMorph (version 5.0). Numerical data were exported to Excel (version X) and plotted using SigmaPlot (version 7.0). For SEM, tissue segments were dried in hexamethyldisilazane, sputter coated with 100- $\AA$ gold-palladium, and then imaged with an ISI SS40 at $10 \mathrm{kV}$ (Yale University) or a JEOL JSM-840 at 15 kV (Marine Biological Laboratory, Woods Hole, MA). Prints from the ISI SS40 were digitized at $600 \mathrm{dpi}$; images from the JEOL JSM-840 were digitally recorded at $150 \mathrm{dpi}(3360 \times 2600)$.

\section{SDS-PAGE and Immunoblotting}

Protein fractions were analyzed with SDS-PAGE by using 5-20\% gradien gels. Protein concentrations were assessed with the bicinchoninic acid assay (Pierce Chemical, Rockford, IL). For immunoblotting, gels were transferred to nitrocellulose $(85 \mathrm{~V}, 3.5 \mathrm{~h})$ at $4^{\circ} \mathrm{C}$. Immunogens were visualized using the enhanced chemiluminescence method (Amersham Biosciences, Piscataway, NJ). Primary antibodies used for immunoblots in these studies include (used at 1:1000 unless otherwise noted) the following: anti-Myo1a (Skowron et al., 1998); anti-CaM (monoclonal antibody [mAb]; Upstate Biotechnology, Lake Placid, NY); anti-Myole (Skowron et al., 1998), anti-Myo2a (Biomedical Technologies, Cambridge, MA); anti-Myo5 (Espindola et al., 1996); anti-Myo6 (Hasson and Mooseker, 1994); anti-Myo10 (gift from R. Cheney, University of North Carolina, Chapel Hill, NC); CX-1 (1:500) (Carboni et al., 1988); antivillin (mAb; AMAC, Westbrook, Maine); anti-fimbrin (Heintzelman and Mooseker, 1990a); anti-spectrin (Pearl et al., 1984); anti-WSCK, anti-cytokeratin (CK)8, and anti-CK18 (mAb; Abcam, Cambridge, MA); anti-adaptin $\beta$ and anti-clathrin (BD Transduction Laboratories, Lexington, KY); anti-rat SI (gift from A. Quaroni, Cornell University, Ithaca, NY); anti-AP (1:2000; SigmaAldrich, St. Louis, MO); anti-galectin-4 (Gal4) (R\&D Systems, Minneapolis, $\mathrm{MN}$ ); and anti-Myo1c (gift from P. Gillespie, OHSU).

\section{RESULTS}

\section{Development of the Myo1a KO Mouse}

Conventional ES cell/recombination protocols were used to replace the first three exons of the Myola gene with a cassette conferring resistance to neomycin (Figure 1A). Proper positioning of the $\mathrm{KO}$ cassette was confirmed with genomic PCR by using primers that flanked the targeted region (Figure $1 \mathrm{~A}, \mathrm{~F} 1$ and $\mathrm{B} 1$ ). The resulting $\mathrm{KO}$ product demonstrated the expected 2311-base pair insertion and the introduction of two new EcoRI sites (Figure 1B). Ablation of the Myo1a gene product in homozygote animals was confirmed by immunoblot analysis of whole gut, mucosal homogenate, and crude $\mathrm{BB}$ fractions by using a polyclonal directed against the TH1 domain of Myo1a (Figure 1C). Immunostaining of KO duodenal sections with the same antibody revealed the expected lack of signal in the BB region (Figure 1D, arrowheads).

\section{Myo1a KO Mice Exhibit No Overt Phenotype}

Homozygous $\mathrm{KO}$ mice demonstrated no overt phenotype at the whole animal level (Figure 2A). With respect to growth rate, weight gain, rate of stool production, and consistency (Figure 2B), KO mice seemed no different from WT. Moreover, levels of physical activity, reproductive rate, and litter size also were normal. Although a recent report suggests that mutations in Myola may result in deafness in humans (Donaudy et al., 2003), KO animals responded well in a startle test and performed normally in balance beam trials, suggesting that hearing and vestibular function were unperturbed.

\section{Intestinal Histology}

To assess irregularities in the morphology of $\mathrm{KO}$ small intestine, duodenal sections were embedded in paraffin and stained with $\mathrm{H} \& \mathrm{E}$. $\mathrm{KO}$ sections revealed no obvious perturbations in villus or crypt organization (Figure 2C). Both mucosa and submucosa seemed normal; no defects in enterocyte structure were apparent at this level of resolution. However, measurements of villus length in the $\mathrm{KO}$ revealed a small but statistically significant decrease relative to WT $(\mathrm{KO}, 440 \pm 57 \mu \mathrm{m}, \mathrm{n}=144$ villi vs. WT, $468 \pm 46 \mu \mathrm{m}, \mathrm{n}=$ 144 villi; $\mathrm{p}<0.0005)$. Villus shortening is a hallmark of intestinal inflammation (Kuisma et al., 2001) and can be linked to higher levels of apoptosis at villus tips. Consistent with this, TUNEL assays demonstrated that KO sections possessed significantly higher numbers of apoptotic cells relative to WT (Figure 2D). High-magnification views of $\mathrm{KO}$ villus structure also revealed that TUNEL signal was not restricted to villus tips where continuous cell shedding normally occurs (Figure 2E). Instead, the lamina propria, which is not normally apoptotic, also demonstrated bright TUNEL staining (Figure 2E, insets). To assess duodenal morphology with higher resolution, we used SEM. Most villi in these micrographs demonstrated normal morphology; yet occasional villi with obvious thickening and fractured tips were also observed (Figure 2F). Thus, although KO mice do not exhibit significant phenotypes at the whole animal level, this evidence indicates that the $\mathrm{KO}$ small intestine may persist in a state of elevated stress.

\section{Ultrastructural Analyses}

Apical Membrane Defects in KO Enterocytes. Our initial ultrastructural analyses focused on assessing the topology and integrity of the apical membrane. Low-magnification SEM revealed that the surfaces of villi from $\mathrm{KO}$ duodenum were much "rougher" than WT (Figure 3, A and B). The perceived roughness at this magnification is due to the nearly ubiquitous presence of membrane extrusions at the tips of MV, as well as greater disorder in MV length and packing (see below). Although absent in WT, these irregularities were present on nearly every cell in a given field and observed along the full length of the villus. Apical surface defects similar to those observed in the duodenum also were found in the ileum (Figure 4, D and E), but no obvious flaws were observed in the colon (Figure $4 \mathrm{~F}$ ). The confinement of these phenotypes to the small intestine is consistent with the expression profile of Myola along the gut; immunoblots showed that Myola expression levels in colon are much lower than duodenum or ileum (Figure 4A, inset).

Closer examination of the apical surface in $\mathrm{KO}$ duodenum also revealed very large, pleomorphic extrusions of membrane emanating from the tips of individual MV (see asterisks in Figure 5A [for mild] and B [for severe]). We speculate that these extrusions may represent precursors to the larger, more dramatic herniations that were observed at a lower frequency but encompassed multiple MV (Figure 5C, brackets). TEM showed that these larger herniations contained cytoplasm (Figure 5, D-M) and often retained MV actin bundles associated with the plasma membrane at the periphery (Figure 5E, arrowheads).

KO MV Are Susceptible to Core Solation. TEM and SEM of Myo1A KO enterocytes also revealed extensive vesiculation, similar in appearance to isolated MV (Mooseker et al., 1983) and BBs (Burgess and Prum, 1982) treated with elevated $\mathrm{Ca}^{2+}$ (Figure 6). The "sausage link" appearance of MV in these BBs (Figure 6B, arrowheads) is due to the lack of an 

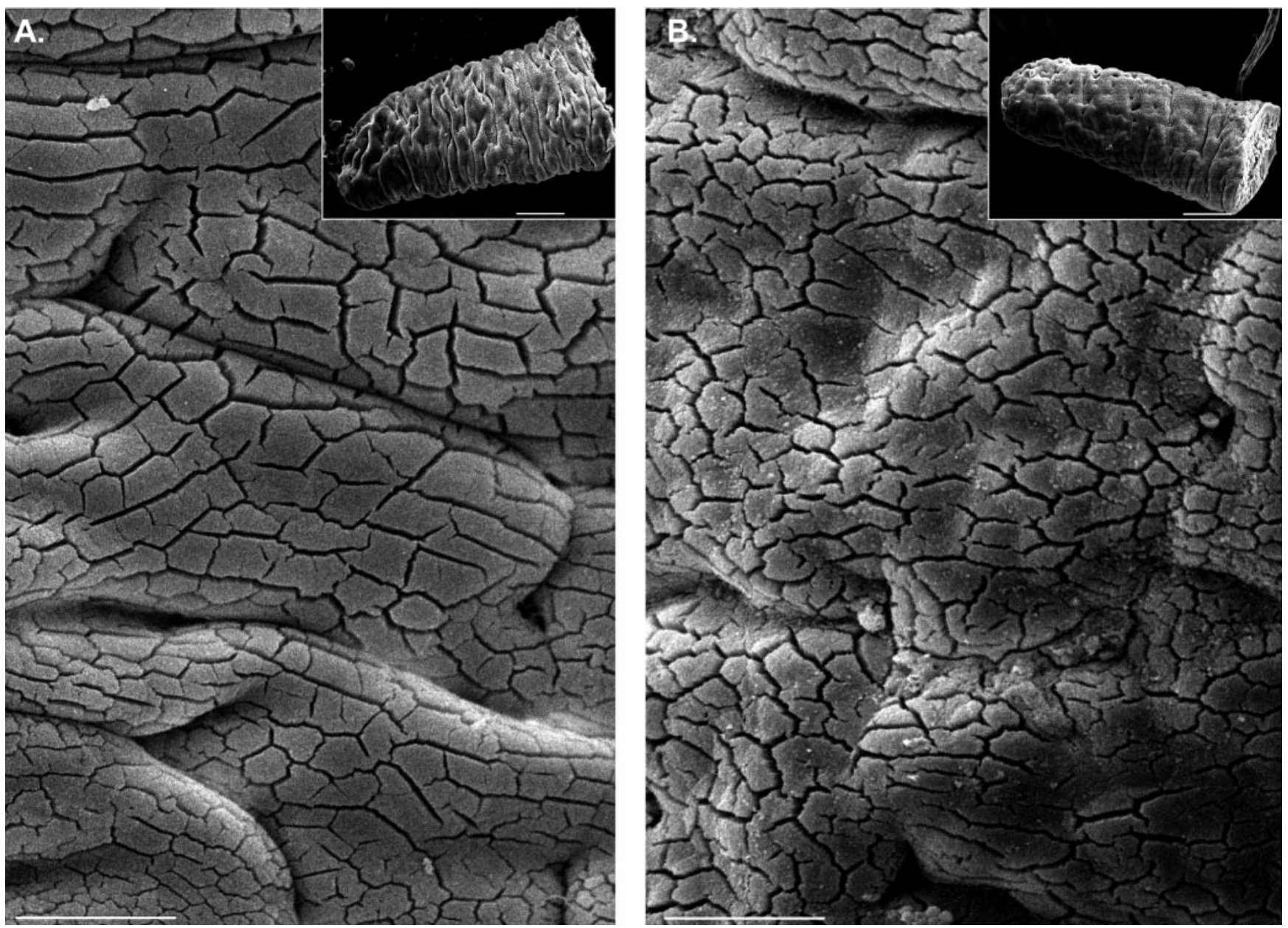

Figure 3. Low-magnification SEM of WT (A) and KO (B) villus surfaces. KO villus surfaces exhibit a general roughness due to irregularities in the apical membrane. Bars, $25 \mu \mathrm{m}$. The larger surface "cracks" apparent in these micrographs were formed as a result of the MV clumping that takes place during sample preparation (see Materials and Methods); they do not represent ultrastructural damage. Clumping enabled lateral views of microvilli at higher magnifications (see Figure 4). Insets show whole villi. Bars, $100 \mu \mathrm{m}$.

intact actin core, likely the result of $\mathrm{Ca}^{2+}$-induced, villindependent severing (Mooseker, 1985). The presence of extensive vesiculation, which was absent in WT preparations, suggests that $\mathrm{KO}$ BBs may have difficulties buffering the $\mathrm{Ca}^{2+}$ fluxes normally encountered during ad libitum feeding conditions. Compositional changes in KO BBs (discussed below) provide an explanation for this lost buffering capacity.

Structural Disarray in KO BBs. KO BBs exhibited a pronounced lack of overall organization. Low-magnification TEM on whole gut sections showed that KO enterocytes lacked the highly ordered, tightly packed arrays of MV (Figure 7, D-F) that were obviously present in WT BBs (Figure 7, A-C). Although WT BBs are packed so tightly that free space between MV is eliminated, $\mathrm{KO}$ BBs exhibit a striking looseness and clearly discernible gaps between individual MV. These differences were obvious in longitudinal sections (compare Figure 7, B and E), but cross-sectional views of the BB (compare Figure 7, C and F) allowed us to quantify this defect by assessing the packing angle $(\gamma)$ between adjacent MV. WT MV were packed at a mean $\gamma$ of $59.0 \pm 8.0^{\circ}$ (Figure $7 \mathrm{G}, \mathrm{WT}$ ), very close to the ideal $60^{\circ}$ that would be expected for the hexagonal pattern created by the tight packing of perfect cylinders. However, the mean $\gamma$ between $\mathrm{KO}$ MV was significantly higher and exhibited a much broader range $\left(67.5 \pm 16.8^{\circ}\right)$, reflecting a loss of hexagonal packing (Figure 7G, KO). Cross-sectional views also allowed us to make measurements of MV and actin core cross-sectional area. These observations revealed that the distance between the actin core and apical membrane (calculated from the difference between these two areas) is significantly reduced in the KO MV (Figure 7H), presumably due to the absence of Myola lateral bridges (only visible in isolated BBs; see below). Although we observed no significant differences in actin core cross sectional area (which reflects the number of bundled actin filaments), an accurate assessment of this parameter was precluded by the fact that it varies as a function of MV length (our unpublished observations), which cannot be determined in crosssectional views. However, in longitudinal sections, $\mathrm{KO} \mathrm{MV}$ did seem more variable in length, even within a single BB (compare Figure 7, B and E). WT MV length was relatively constant within a single cell with a SD $<5 \%$ of the mean length (Figure 7I, WT), whereas $\mathrm{KO}$ variability was significantly higher ( $\sim 10 \%$ of mean length; Figure $7 \mathrm{I}, \mathrm{KO})$. In addition to overall MV disarray, the KO terminal web (TW) region seemed highly disorganized with rootlets of variable length and a poorly defined connective meshwork that 

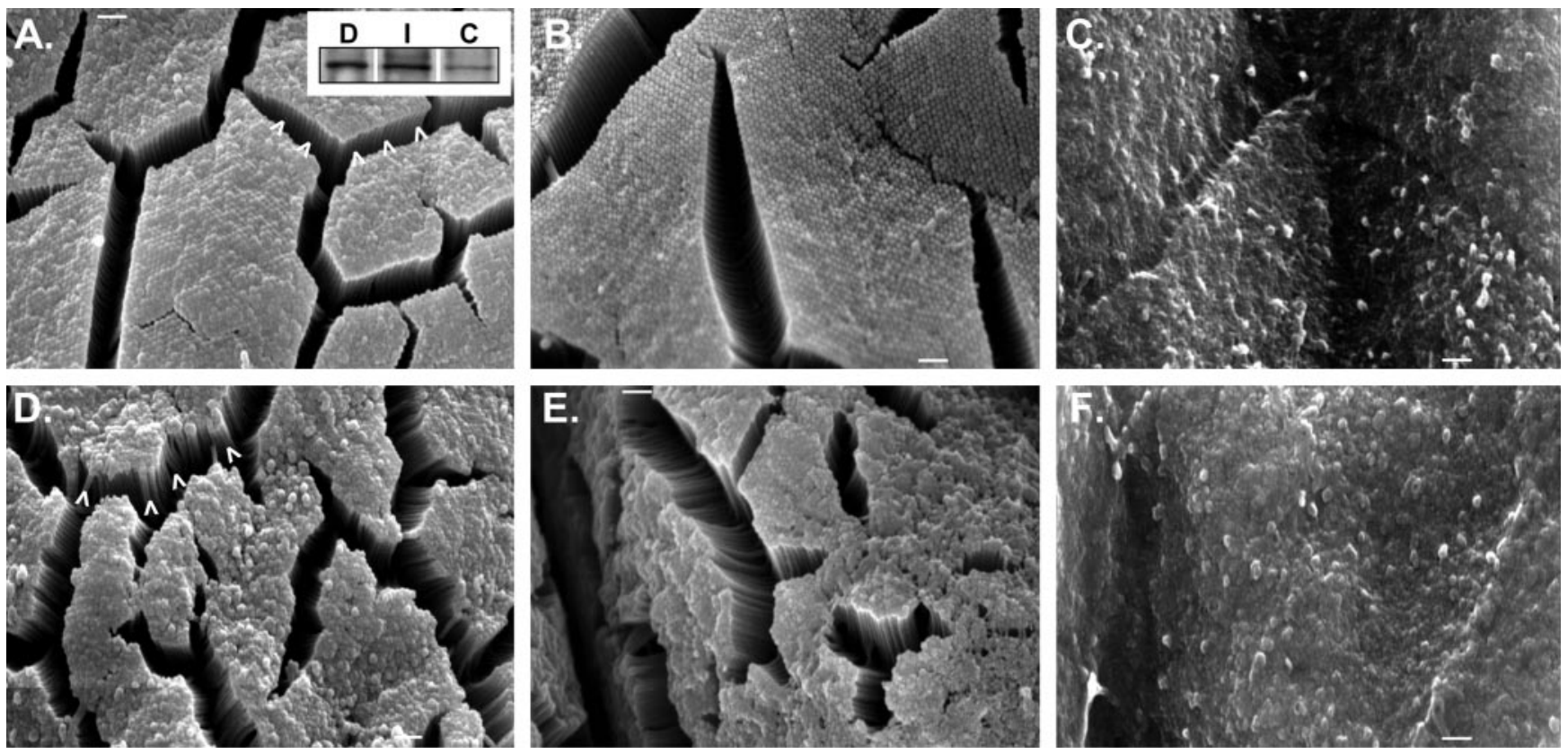

Figure 4. SEM of WT (A-C) and KO (D-F) enterocyte surfaces sampled at three positions along the gut axis: duodenum (A and D), ileum (B and E), and colon (C and F). Apical surface perturbations are confined to the small intestine (duodenum and ileum) in KO mice. Disarray in MV packing is evident in lateral views of KO BBs (compare arrowheads in A and D). Bars, $1 \mu \mathrm{m}$. Inset in A shows anti-Myola immunoblots on whole cell homogenates prepared from WT duodenum (D), ileum (I), or colon (C) and loaded for equal total protein.

failed to exclude cytosolic material (compare Figure 7, B and E). Although we did attempt to quantify the apparent disarray, in general $\mathrm{KO}$ rootlets were much more difficult to distinguish, a fact that confounded angle and length measurements. Together, these ultrastructural observations suggest that Myola plays a critical role in maintaining the exquisite organization and tight packing of MV; hallmark features of the enterocyte BB.

\section{Compositional Analyses}

Lateral Bridges Are Missing in KO MV. Although we were able to isolate $\mathrm{KO}$ BBs with apical membranes and actin cytoskeleton intact (Figure 8, H and I), they appeared more disheveled and less refractile compared with WT BBs (Figure $8, \mathrm{~A}$ and $\mathrm{B}$ ). This appearance is most likely an in vitro manifestation of the structural disarray described in our ultrastructural analyses of whole gut sections (see above). Isolated BBs also were incubated in buffer containing high levels of $\mathrm{Mg}^{2+}$ to enhance the visualization of lateral bridges (Mooseker and Tilney, 1975). The prominent bridges that were clearly visible in WT MV were absent in the KO (compare Figure 8, C and J). This absence resulted in apical membranes that were collapsed onto the actin core surface and extruded from MV tips. In combination with our ultrastructural analyses (see above), these results clearly indicate that Myola lateral bridges are required to maintain proper spacing between the MV actin core and the apical membrane.

Based on the results of in vitro motility studies (Mooseker and Cheney, 1995), it has been assumed that Myola bridges exert an outward (plus-end-directed) force on the MV membrane, but direct evidence for this has been lacking. We observed that the addition of $1 \mathrm{mM}$ ATP to WT BBs induced both myosin-IIa (Myo2)-dependent TW contraction (Mooseker, 1985) and extrusion of apical membranes from the underlying actin cytoskeleton (Figures 8, D-G). In WT BBs, any membrane shedding or damage that occurs in the presence of ATP could result from dissociation of Myola tethers or active Myoladependent membrane movements. However, only TW contraction was observed in $\mathrm{KO}$ (Figures $8, \mathrm{~K}-\mathrm{N}$ ), providing the first direct evidence that Myola can exert concerted outward force on the BB membrane.

KO BBs Are Deficient in Membrane and Cytoskeletal Components. SDS-PAGE analysis of isolated BB fractions from $\mathrm{KO}$ indicated that the majority of proteins (visualized with Coomassie Blue) are present at levels comparable with WT (Figure 9A). However, prominent bands were missing at $\sim 110$ and $\sim 20 \mathrm{kDa}$; immunoblots confirmed that these correspond to Myola heavy chain and its light chain, CaM, respectively (Figure 9A). The latter finding provides a biochemical basis for the vesiculation defects that were apparent in our ultrastructural analyses, because CaM serves as the primary $\mathrm{Ca}^{2+}$ buffer in this region of the enterocyte.

Isolated $\mathrm{BBs}$ from $\mathrm{KO}$ mice also were subject to an extensive immunoblot screen with probes directed against a number of BB components (Figure 9 and Table 1). It should be noted that $\mathrm{BBs}$ from $\mathrm{KO}$ enterocytes exhibiting the most extreme perturbations (e.g., herniations and vesiculation) are probably lost during the initial mucosal homogenization (i.e., purification selects for the most robust BBs). Thus, differences between WT and $\mathrm{KO}$ BB preparations reported here may actually underrepresent differences that are present in vivo. Immunoblots revealed that many BB cytoskeletal proteins exhibited normal levels of association in KO preparations: villin and fimbrin (MV core components), myosin-5a (Myo5), Myo2, and spectrin (TW components) all exhibited WT levels (Figure 9B). In contrast, the lipid raftassociated proteins sucrase-isomaltase (SI) and Gal4 were both reduced in $\mathrm{KO}$, whereas AP was unchanged (Figure 9B and Table 1). Other cytoskeletal components that were de- 

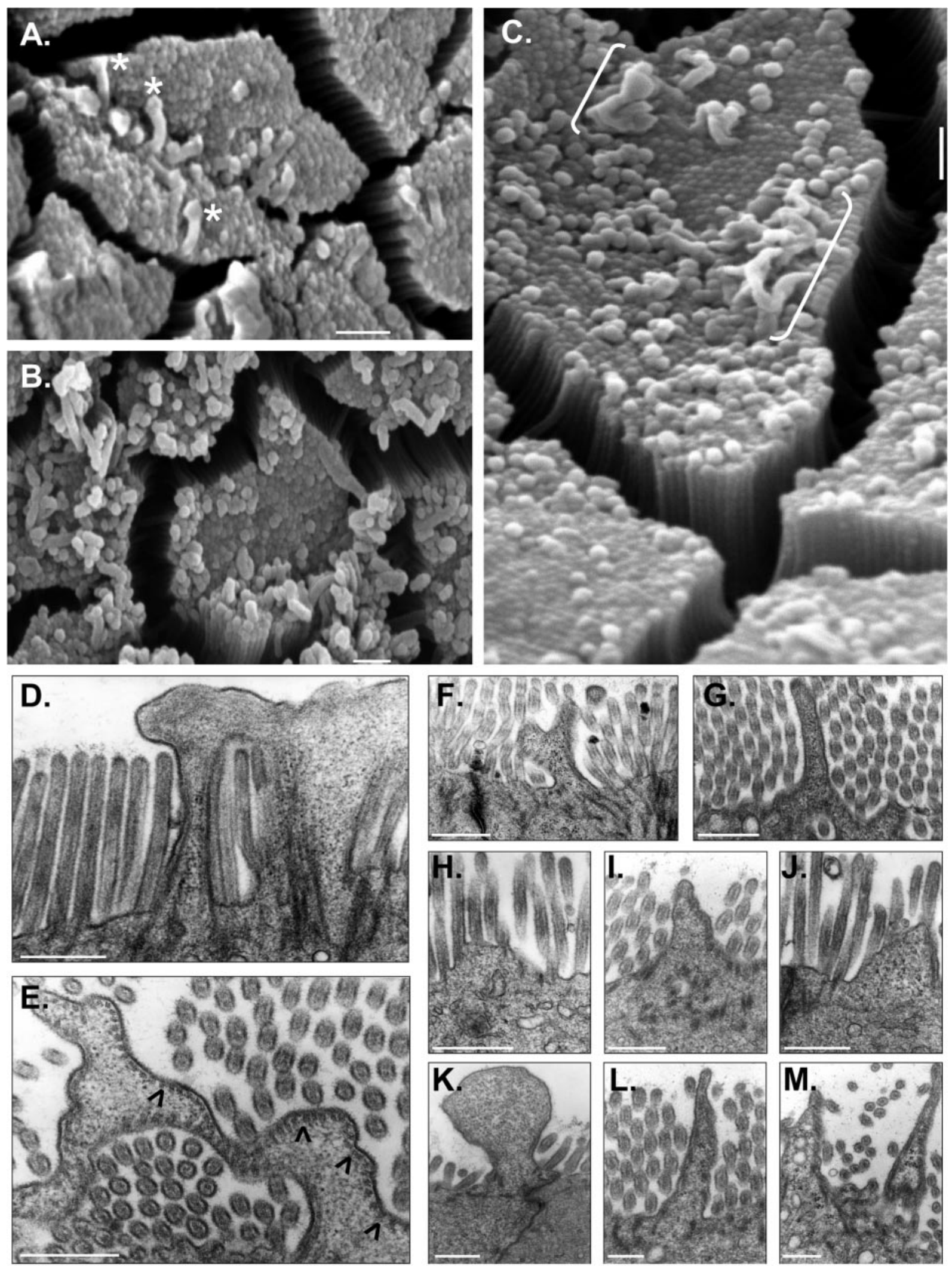

Figure 5. SEM (A-C) and TEM (D-M) of apical membrane defects in KO enterocytes. Mild (A) and severe (B and C) cases of membrane extrusion from MV tips are shown. White asterisks (A) mark membrane extrusions that seem to emanate from individual MV. White brackets (C) denote larger herniations that seem to encompass multiple MV. Cross-sections obtained with TEM (D and E) show that larger membrane herniations are filled with cytosol; black arrowheads in E mark regions where actin core-apical membrane associations are retained. Other examples (F-M) demonstrate the diversity of apical membrane herniations observed in KO BBs. Bars, $1 \mu \mathrm{m}(\mathrm{A}-\mathrm{C})$ and $0.5 \mu \mathrm{m}(\mathrm{D}-\mathrm{M})$. 

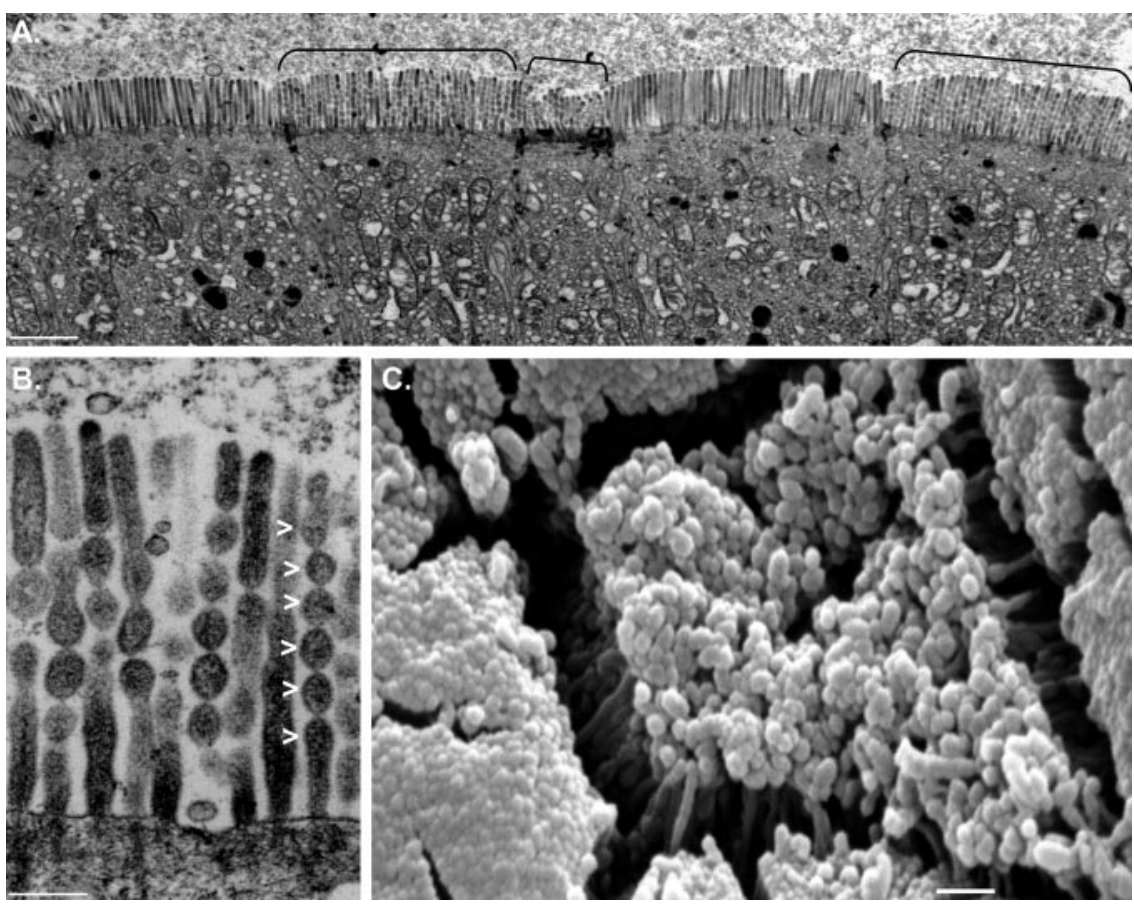

Figure 6. (A) Extensive vesiculation of BBs on multiple adjacent enterocytes (see black brackets). (B) Higher magnification reveals the sausage link appearance that is characteristic of villin-induced actin core severing (see white arrowheads). (C) SEM shows an en face view of an enterocyte with an extensively vesiculated BB. Bars, $2 \mu \mathrm{m}(\mathrm{A})$, $250 \mathrm{~nm}(\mathrm{~B})$, and $1 \mu \mathrm{m}(\mathrm{C})$. ficient in $\mathrm{KO} \mathrm{BB}$ preparations include another class I myosin, myosin-1e (Myo1e), and myosin-VI (Myo6), a motor implicated in endocytosis. A slight reduction in the clathrin adaptor protein, adaptin $\beta$, was also observed and may be related to the loss of Myo6 from these fractions (Figure 9B and Table 1). Immunoblots of whole cell homogenates showed that the reductions in CaM, Myole, and Myo6 were not the result of large changes in cellular expression levels, but instead due to the redistribution of these components in $\mathrm{KO}$ enterocytes (Figure 9C).

Consistent with our immunoblot analyses, immunostaining of duodenal tissue sections for SI revealed reduced staining intensity in the BB and low, but detectable, levels of basolateral signal not seen in WT (Figure 9D). Moreover, the TW localization of Myo6 normally observed in WT was absent in the $\mathrm{KO}$, confirming that the reduction observed in immunoblots was not due to a differential loss during $\mathrm{KO}$ $\mathrm{BB}$ isolation (Figure 9D). Other BB components that were screened in $\mathrm{KO}$ duodenal tissue sections but that demonstrated no difference in localization relative to WT include actin, villin, fimbrin, $\mathrm{Ca}^{2+}$ transporter-1, and myosin-10 (our unpublished data).

In contrast to the numerous deficiencies in $\mathrm{KO}$ BBs discussed above, immunoblots of $\mathrm{KO}$ BBs demonstrated a striking increase in the intermediate filament protein pair CK8 and CK18 (Figure 9B and Table 1). Immunostaining of KO duodenal tissue sections with a WSCK polyclonal (recognizes both CK8 and CK18) showed much higher levels of signal in the $\mathrm{BB}$ region of $\mathrm{KO}$ enterocytes (Figure 9D). Because immunoblots of whole cell homogenates with the same antibody revealed no change in total cellular cytokeratin (Figure 9C), these elevated levels are likely the result of redistribution within $\mathrm{KO}$ enterocytes.

\section{Myosin-1c Is Ectopically Recruited to the BB in $\mathrm{KO}$ Enterocytes}

One of the most striking revelations from our immunoblot screen was the ectopic recruitment of Myo1c into KO BBs
(Figure 10A). Fractionation of $\mathrm{KO}$ enterocytes revealed that recruitment to the $\mathrm{BB}$ was fueled by a loss of Myo1c from other membrane compartments (most obvious when comparing low-speed pellet and supernatant fractions; Figure 10A). KO duodenal sections stained for Myo1c revealed the same profound redistribution. In WT enterocytes, this motor preferentially associated with basolateral membranes and exhibited very little staining in the apical domain (Figure $10 \mathrm{~B}$, arrowheads). In $\mathrm{KO}$ sections, the pronounced basolateral signal was lost and Myo1c was instead found in the BB (Figure 10B, arrowheads).

Given our previous studies establishing Myola as a lipid raft component, required for the proper localization of SI (Tyska and Mooseker, 2004), we sought to determine whether Myo1c also was recruited to raft fractions in $\mathrm{KO}$ BBs. To this end, a detergent-insoluble raft fraction was produced from equal quantities of purified WT and KO BBs (see Materials and Methods). Density gradient analysis of this fraction revealed that the lowest density (raft-containing) fraction from $\mathrm{KO}$ BBs was in fact enriched with Myolc relative to the WT fraction (Figure 10E). This first fraction also contained SI, but densitometry of the blot film images revealed that levels were reduced $\sim 20 \%$ compared with WT (Figure 10F). The total amount of SI (the sum of SI signal across the entire gradient) also was reduced by $\sim 30 \%$. Interestingly, much less flocculent material coalesced at the top of KO gradients, indicating a reduced amount of detergent-insoluble lipid (our unpublished data). Consistent with this, the total amount of detergent-insoluble protein liberated from KO BBs (as visualized with Coomassie Blue) also was significantly reduced relative to the WT preparation (Figure 10C), suggesting an overall perturbation in lipid raft stability.

These observations raise the possibility that the loss of Myola function in $\mathrm{KO}$ mice may be rescued by the ectopic recruitment of Myo1c to the BB. However, the ultrastructural and biochemical perturbations described above sug- 

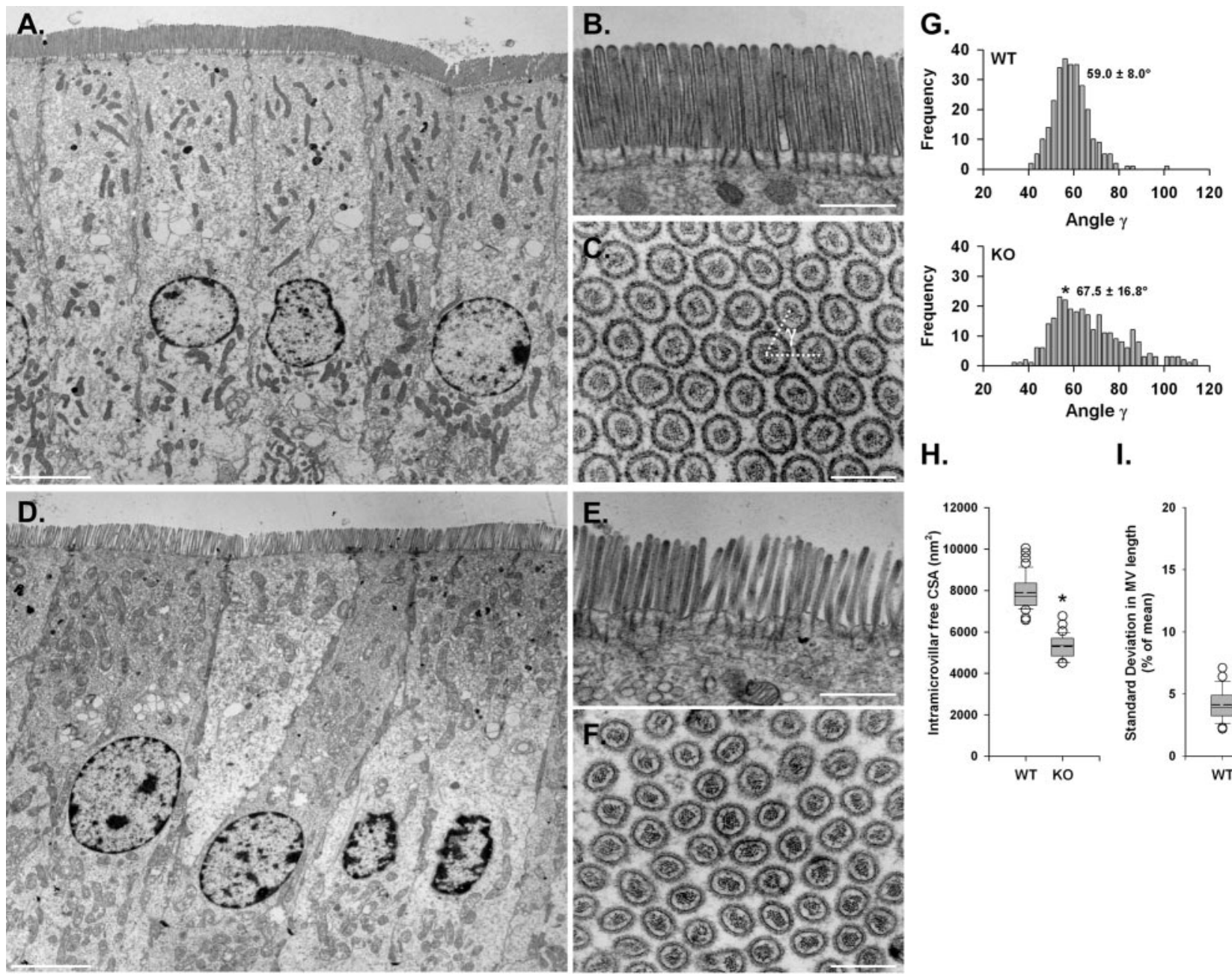

H.

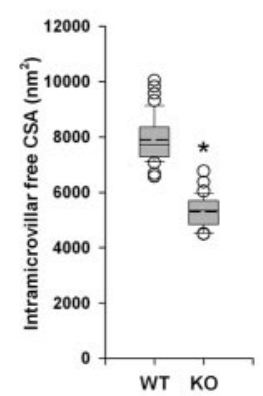

I.

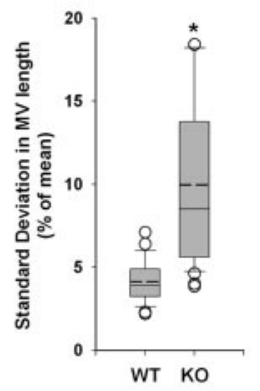

Figure 7. TEM of WT (A-C) and KO (D-F) enterocytes reveals poor organization and loose packing of MV in the absence of Myo1a. TW disruptions are also obvious at higher magnification (compare B and E). Cross sections through WT (C) and KO (F) BBs show significant disarray in MV packing. (G) Mean packing angle ( $\gamma$, see white dashed lines in C) measured from KO cross sections is significantly higher and more variable relative to $\mathrm{WT}\left(\mathrm{WT}, 59.0 \pm 8.0^{\circ}, \mathrm{n}=280 \mathrm{vs}\right.$. $\left.\mathrm{KO}, 67.5 \pm 16.8^{\circ}, \mathrm{n}=286 ;{ }^{*} \mathrm{p}<0.0005\right)$. $(\mathrm{H})$ Amount of free cross-sectional area (CSA) between the MV actin bundle and the apical membrane (calculated as MV CSA - actin core CSA) is significantly reduced in KO MV (WT, $7883 \pm 834 \mathrm{~nm}^{2}, \mathrm{n}=40$ vs. $\mathrm{KO}, 5294 \pm 579 \mathrm{~nm}^{2}, \mathrm{n}=32$; ${ }^{*} \mathrm{p}<0.0005$ ). (I) SD in MV length per cell is significantly greater in KO (WT, $4.1 \pm 1.2 \%, \mathrm{n}=345 \mathrm{MV}$ from 23 cells vs. KO, $10.0 \pm 5.0 \%, \mathrm{n}=435 \mathrm{MV}$ from 29 cells; $\left.{ }^{*} \mathrm{p}<0.0005\right)$. Data in $\mathrm{H}$ and I are presented as box-plots as described in the Figure 2 legend. Bars, $5 \mu \mathrm{m}$ (A and D), $1 \mu \mathrm{m}$ (B and E), and $200 \mathrm{~nm}$ (C and F).

gest that this rescue is only partial, not complete. Reasons for this are discussed in more detail below.

\section{DISCUSSION}

Our multilevel phenotypic characterization of Myola KO mice has revealed a surprisingly diverse array of cellular level perturbations, ranging from defects in apical membrane topology to aberrant $\mathrm{BB}$ organization and composition. However, the impact of these perturbations is not immediately obvious at the whole animal level, begging the question: How does gross level physiological function remain unaffected in the presence of these BB level defects? Within the small intestine, crypt regions found at the base of villi provide a continuous supply of newborn enterocytes that migrate up toward the villus tip as a continuous sheet of cells (normally an $\sim 48$-h trip). This process of regenerative renewal (which may even be accelerated in the $\mathrm{KO}$; see below) provides the intestine with an immense capacity for accommodating mucosal damage (Mammen and Matthews, 2003). In $\mathrm{KO}$ animals, this capacity may serve as a protective mechanism, potentially blunting the whole animal response to cellular level defects.

Despite the lack of a whole animal phenotype, evidence from our histological analyses suggests that $\mathrm{KO}$ small intestine may persist in a state of elevated stress. For example, enterocytes in $\mathrm{KO}$ duodenum demonstrate significantly higher levels of apoptosis (mucosal and submucosal) and accompanying reductions in villus height. These responses, which represent hallmark symptoms of mucosal stress, also are associated with malnutrition (Estivariz et al., 1998), intestinal inflammation (Kuisma et al., 2001), and intestinal infection (Sasahara et al., 2003). Another potential sign of mucosal stress relates to the higher levels of CK8 and CK18 found in KO BBs. This intermediate filament dimer pair has been implicated in a wide range of stress-related functions, including the regulation of apoptotic signaling pathways (Gilbert et al., 2001) and the mechanical stabilization of cell 

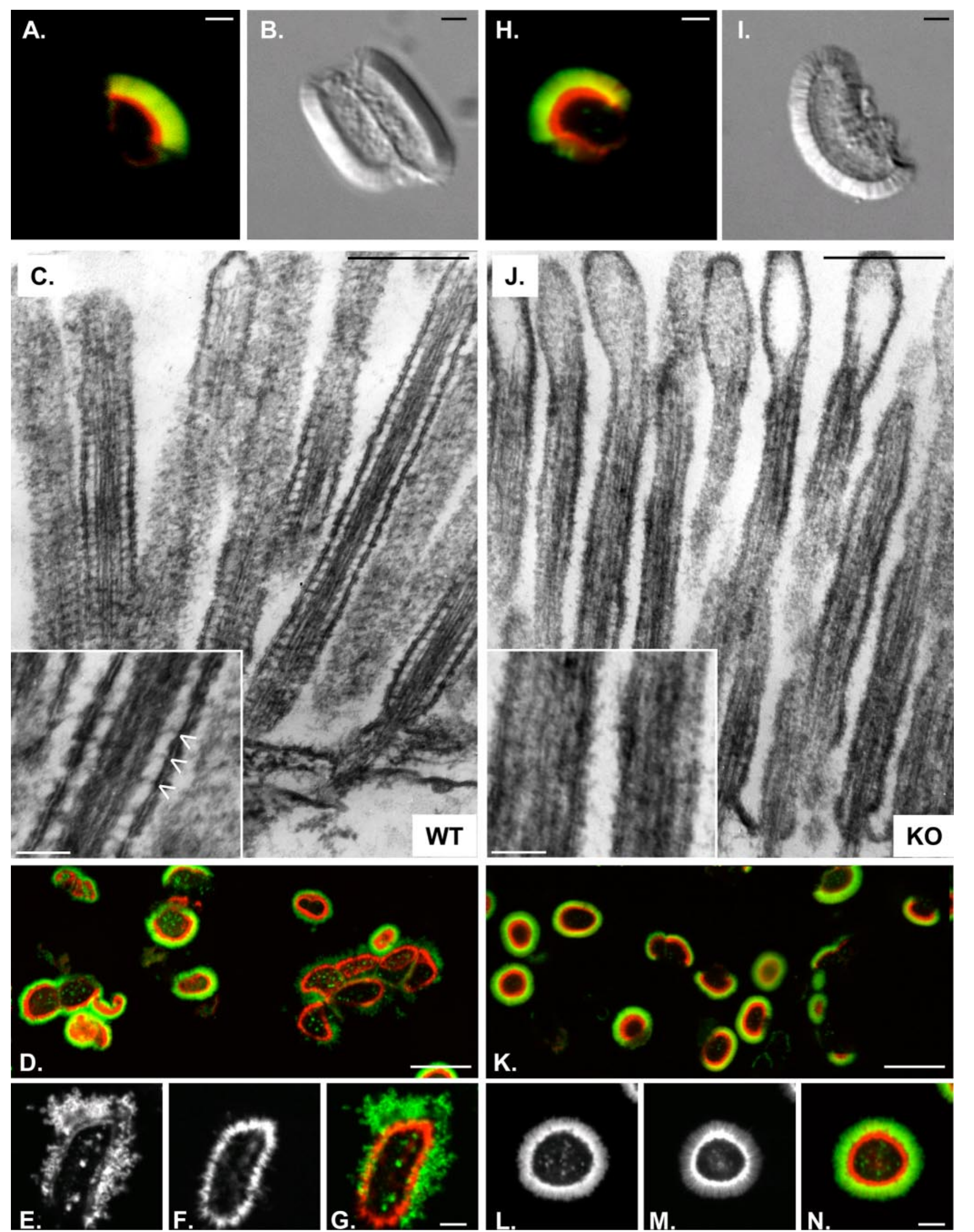
A.

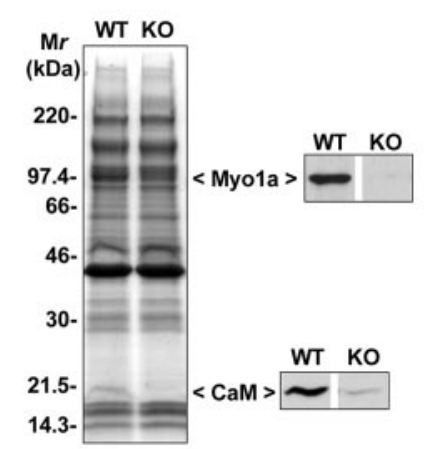

B.

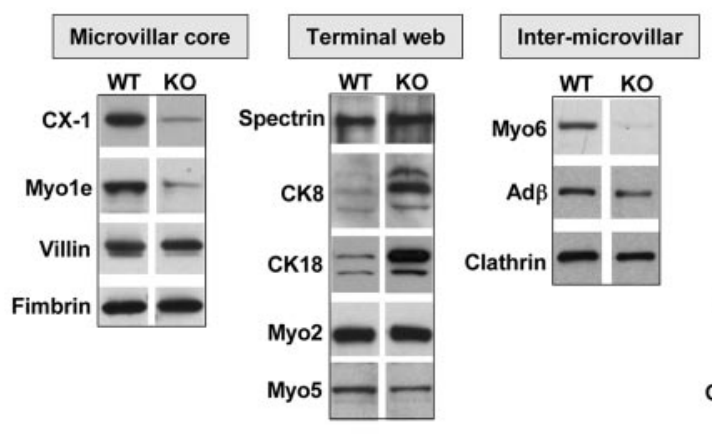

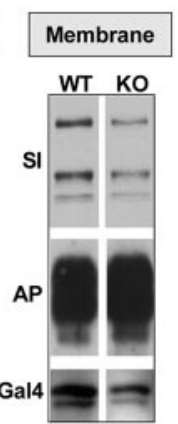

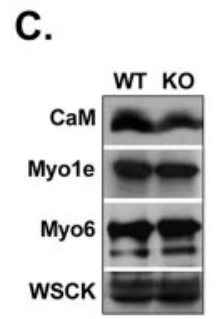

D.
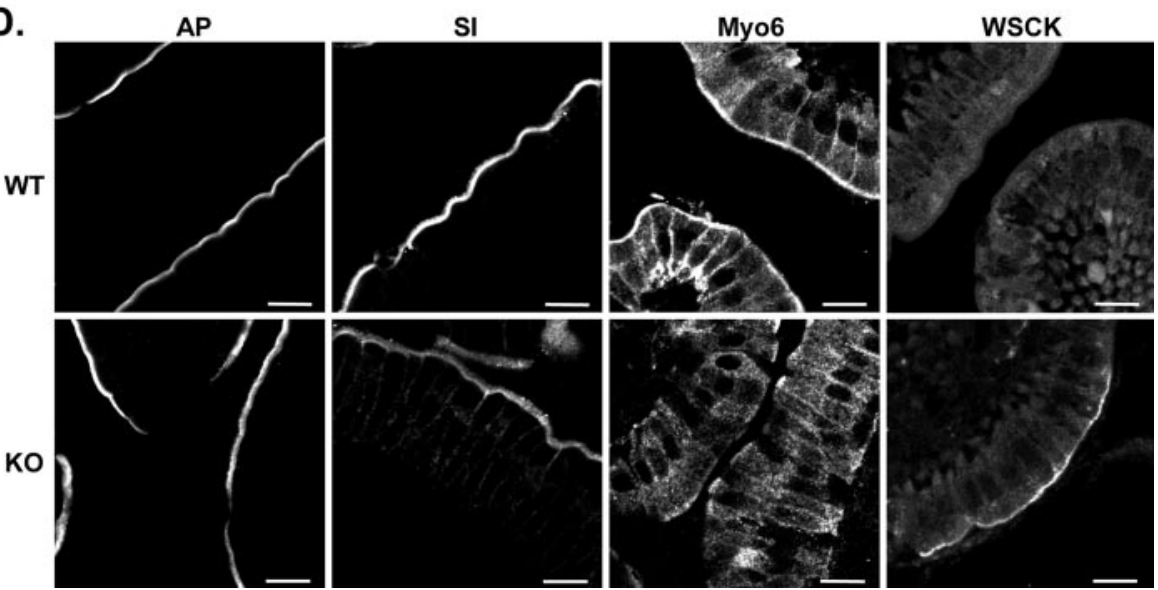

Figure 9. (A) SDS-PAGE analysis of BBs isolated from WT and KO small intestine (loaded for equal total protein). Immunoblots confirm that the missing bands at $\sim 110$ and $\sim 20 \mathrm{kDa}$ correspond to Myola and its light chain, CaM. (B) Immunoblot screen of isolated BBs (loaded for equal total protein) reveals that several components are lost in $\mathrm{KO}$, including Myo1e, Myo6, SI, and Gal4. CK8 and 18 are significantly elevated relative WT. (C) Immunoblots of whole cell homogenates show that total cellular levels of CaM, Myole, Myo6, and the cytokeratins (with WSCK) are no different in KO. SI and Gal4 were not readily detectable in these dilute fractions. (D) Immunostaining of KO duodenal sections shows the redistributions (relative to WT) of SI, Myo6, and the cytokeratins, but not AP. Bars, $10 \mu \mathrm{m}$.

morphology during osmotic stress (Ding et al., 1998). All of these symptoms together may reflect a physiological attempt to maintain homeostasis in spite of a compromised mucosa.

Throughout the course of these investigations, Myola KO mice experienced a highly regular and buffered existence, e.g., ad libitum food and water and no environmental stress. However, because mucosal repair mechanisms are already taxed in the $\mathrm{KO}$ under these mild conditions (as suggested by higher level apoptosis and villus shortening), it remains possible that overt phenotypes may be induced through exposure to nutritional stress or insults from chemical agents or bacterial pathogens. Ferrary et al. (1999) used such a tact during their investigation of villin null mice. Although villin $\mathrm{KO}$ mice also demonstrated no overt phenotype, inducing acute ulcerative colitis with dextran sodium sulfate (DSS) (Okayasu et al., 1990) revealed a pronounced inability to cope with mucosal damage that was manifest in an approximately twofold higher probability of death. We also attempted to induce mucosal damage with DSS in Myo1a KO mice, but preliminary experiments demonstrated no significant difference in mortality rate relative to WT mice. This is likely due to the fact that Myola expression levels in the colon (the primary recipient of DSS-induced damage) are very low (whereas villin is highly expressed in both small and large intestine). Agents that have more pronounced effects on the small intestine, such as methotrexate (Taminiau et al., 1980) or Escherichia coli lipopolysaccharide (Caradonna et al., 2000), should enable us to produce obvious physiological consequences.

Ultrastructural analyses of $\mathrm{KO}$ enterocytes revealed a striking assortment of defects in apical membrane topology. These ranged from small membrane extrusions that were confined to single MV, to much larger herniations that en-

Figure 8. Fluorescence (A and $\mathrm{H}$ ) and differential interference contrast (B and I) microscopy of BBs isolated from WT (A and B) and KO (H and I) mice. Although $\mathrm{KO}$ BBs were isolated with membrane (green, ConA) and actin cytoskeleton (red, phalloidin) intact $(\mathrm{H})$, they seem more disheveled (I) relative to WT BBs from the same preparation. (C and J) TEM on isolated BBs incubated in high $\mathrm{Mg}^{2+}$; the lateral bridges that are obvious in WT MV (C, inset) are absent in KO (J, inset). Phalloidin (red) stabilized WT (D-G) and KO (K-N) BBs were fixed and labeled with ConA (green) $15 \mathrm{~min}$ after addition of $1 \mathrm{mM}$ ATP. Higher magnification reveals that ATP-induced membrane shedding is evident in WT (E, ConA; F, phalloidin; G, overlay) but not $\mathrm{KO}$ (L, ConA; M, phalloidin; N, Overlay). Bars are $1 \mu \mathrm{m}(\mathrm{A}, \mathrm{B}, \mathrm{H}$, and I), $250 \mathrm{~nm}$ (C and J), $50 \mathrm{~nm}$ (insets in C and $\mathrm{J}), 10 \mu \mathrm{m}(\mathrm{D}$ and $\mathrm{K})$, and $2 \mu \mathrm{m}(\mathrm{E}, \mathrm{F}, \mathrm{G}, \mathrm{L}, \mathrm{M}$, and $\mathrm{N})$. 
Table 1. Summary of compositional changes in $\mathrm{KO} \mathrm{BBs}$

\begin{tabular}{lc}
\hline Region/component & Change relative to WT \\
\hline Membrane & \\
Sucrase isomaltase & $\Downarrow$ \\
Galectin-4 & $\Downarrow$ \\
Alkaline phosphatase & No $\Delta$ \\
Microvillar core & No $\Delta$ \\
Villin & No $\Delta$ \\
Fimbrin & Absent \\
Myosin-1a & $\Uparrow \Uparrow$ \\
Myosin-1c & $\Downarrow \Downarrow$ \\
Myosin-1e & $\Downarrow \Downarrow$ \\
CX-1 & $\Downarrow \Downarrow$ \\
Calmodulin & $\Downarrow \Downarrow$ \\
Intermicrovillar & \\
Myosin-6 & \\
Clathrin & $\Downarrow \Downarrow$ \\
Adaptin- $\beta$ & No $\Delta$ \\
Terminal web & $\Downarrow$ \\
Myosin-2 & \\
Myosin-5 & No $\Delta$ \\
Spectrin & No $\Delta$ \\
Cytokeratin 8 & No $\Delta$ \\
Cytokeratin 18 & $\Uparrow \Uparrow$ \\
\hline
\end{tabular}

No $\Delta$, no change; $\uparrow / \Downarrow$, small increase/decrease in level; and $\Uparrow \Uparrow / \Downarrow \Downarrow$, large increase/decrease in level.

compassed sizable clusters of MV. We also have observed similar defects in the BBs of CL4 and Caco- $2_{\mathrm{BBe}}(\mathrm{BBe})$ cells overexpressing the Myola TH1 domain as a dominant negative (DN) fragment (our unpublished data). In both the cell lines and Myola KO mice, these perturbations may be explained by a loss of membrane-cytoskeleton tethering in BBs that lack a normal complement of Myola. More specifically, these herniations may be the result of a decrease in overall membrane "stiffness," also referred to as apparent membrane tension (Sheetz, 2001). In KO mice, apparent membrane tension may be depressed, resulting in higher susceptibility to membrane deformation. Biophysical measurements on vertebrate cell membranes have shown that the apparent membrane tension has major contributions from membrane-cytoskeleton adhesion (Dai and Sheetz, 1999). Given its striking localization and high concentration in the BB, we propose that Myola is the primary source of membrane-cytoskeleton adhesion, and thus membrane tension, in the MV. A role for vertebrate myosins-I in the maintenance of membrane tension has yet to be confirmed experimentally, but data from lower eukaryotes provide strong support for this model (Dai et al., 1999).

In addition to apical membrane defects, KO BBs demonstrated significant compositional perturbations. In light of these perturbations, it will be of interest to determine whether $\mathrm{KO} \mathrm{BBs}$ experience increased protein turnover and/or the compensatory expression of other BB proteins. With regard to specific perturbations in $\mathrm{KO}$ BBs, CaM, the $\mathrm{Ca}^{2+}$-binding light chain for Myola, was almost completely missing in isolated fractions. Although low levels of residual CaM were detectable in $\mathrm{KO}$ BBs (most likely bound to other unconventional myosins, e.g., Myo1c), these were inadequate for buffering the cytosolic $\mathrm{Ca}^{2+}$ fluxes experienced by $\mathrm{KO}$ mice during ad libitum feeding. This conclusion is based on the frequent appearance of $\mathrm{KO}$ BBs with extensively vesiculated MV; an appearance similar to that produced by villin severing of isolated MV (Mooseker et al., 1983) and BBs
(Burgess and Prum, 1982) in the presence of high $\mathrm{Ca}^{2+}(>10$ $\mu \mathrm{M})$. We predict that exposing $\mathrm{KO}$ animals to conditions that elevate cytosolic $\mathrm{Ca}^{2+}$ levels (e.g., fasting and refeeding, $\mathrm{Ca}^{2+}$ ionophores, and $\mathrm{Ca}^{2+}$ pump inhibitors) should induce $\mathrm{BB}$ damage with even greater frequency and severity.

$\mathrm{KO}$ BBs also were depleted in Myo6, a component of the clathrin-rich TW region. Similar displacement was observed in BBe cells expressing the DN tail domain of Myola (Tyska and Mooseker, 2002b). Myo6, the only minus-end-directed myosin (Wells et al., 1999), has been implicated in several aspects of receptor-mediated endocytosis in polarized cells (Hasson, 2003). One explanation for loss from the KO TW is that Myola may be involved in the delivery of Myo6, or some factor required for the localization of Myo6 to this region. However, several lines of evidence indicate that Myola-dependent apical delivery is unlikely (see Discussion in Tyska and Mooseker, 2004). Likewise, Myola might be involved in stabilizing Myo6 or a receptor for Myo6 within the TW. However, attempts to copurify Myola and Myo6 in a complex with antibodies directed against either motor have proven unsuccessful (our unpublished data). Alternatively, the interaction between Myola and Myo6 may not biochemical but rather mechanical. In the BB, Myola and Myo6 interact with the same actin "track" (the core bundle), but due to their different mechanical polarities, these two motor populations would be expected to exert antagonistic forces (i.e., push against each other). Intriguingly, recent evidence suggests that Myo6 may transition into an anchorlike state in the presence of significant opposing mechanical load (Altman et al., 2004). It follows that loading may influence the amount of time that Myo6 remains strongly bound to actin in a given region of a cell (i.e., its steady-state distribution) and its ability to perform designated functions. Thus, a final possibility is that the force exerted by Myola on the core actin bundle provides a mechanical stimulus that is required for the proper localization of Myo6 within the TW.

The loss of antagonistic mechanical forces in the $\mathrm{KO} \mathrm{BB}$ (due to the absence of both Myo1a and Myo6) also may help explain the general disarray observed in KO BB architecture. Opposing force generators are known to play a critical role in maintaining the symmetry and organization of other cytoskeletal arrays (e.g., mitotic spindle) (Sharp et al., 2000). In the $\mathrm{BB}$, these mechanical forces may play an integral role in establishing and maintaining the high level of structural order observed under normal conditions.

Other proteins missing from $\mathrm{KO}$ BBs include SI and Gal4, both of which have been identified as components of MV lipid rafts (Danielsen and Hansen, 2003); DRMs that may play critical roles in signaling and apical targeting (Brown and London, 1998). Although SI is a type-II integral membrane disaccharidase involved in carbohydrate processing (Hunziker et al., 1986), Gal4 demonstrates lectin-like properties in that it cross-links and stabilizes raft-associated membrane proteins in the exoplasmic leaflet (Braccia et al., 2003). The loss of raft components from BB fractions, and the decreased amount of detergent-insoluble lipid and protein derived from $\mathrm{KO} \mathrm{BBs}$, strongly indicates that Myola is required for maintaining raft stability. This conclusion is supported by our recent studies with BBe cells expressing the DN tail domain of Myola (Tyska and Mooseker, 2004). This work revealed that Myo1a is a component of DRMs enriched in SI, and functions in the retention of this disaccharidase in the MV membrane. However, the defects observed in the DN model system were much more severe than those reported here. In the DN studies, expression of the tail domain abolished SI localization in the BB (Tyska and Mooseker, 2004), but in KO BBs, SI levels are only partially reduced. 
A.

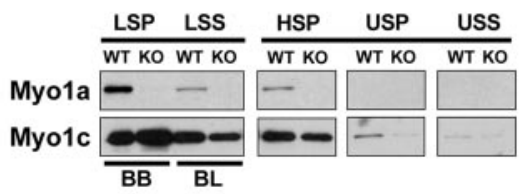

B.
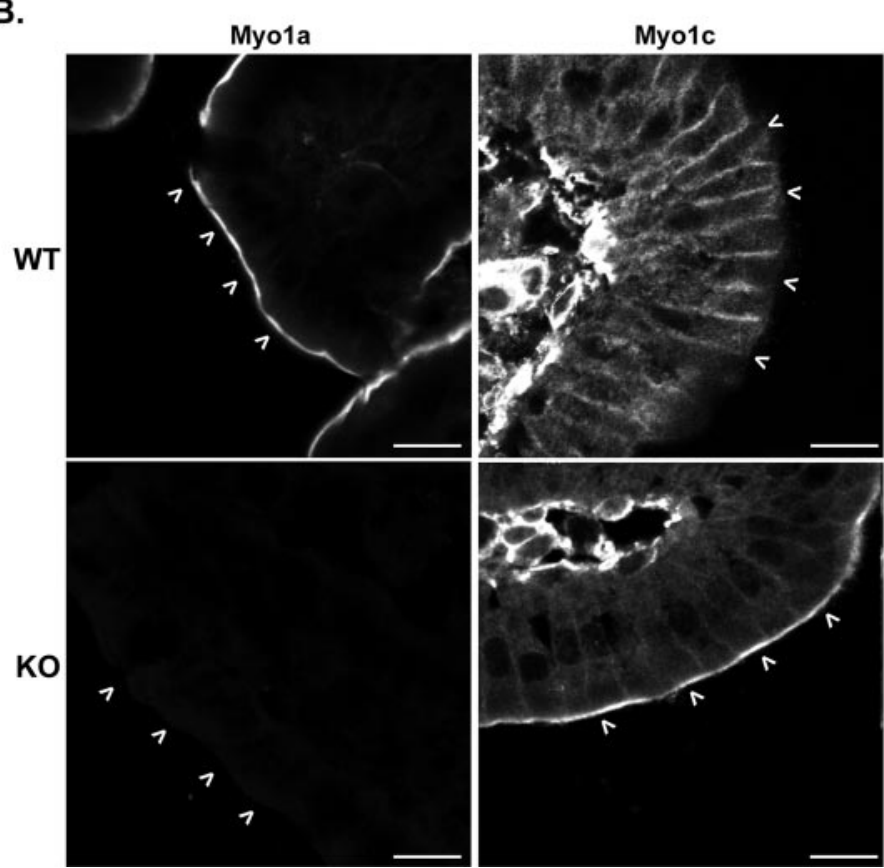

C.

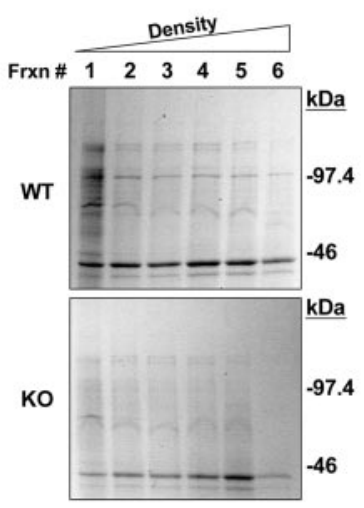

D.

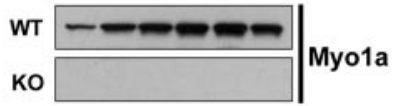

E.

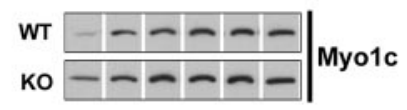

F.

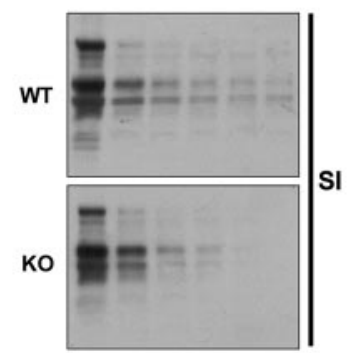

Figure 10. (A) Fractionation of equal amounts (assessed by total protein) of WT and KO enterocytes. In KO, Myo1c is enriched in the BB-rich LSP and lost from the basolateral membrane-rich (BL) LSS, as well as HSP and USP fractions. (B) Immunostaining of WT and KO duodenal sections shows Myo1c redistribution into KO BBs (white arrowheads); the pronounced basolateral staining observed in WT sections is muted; bars are $10 \mu \mathrm{m}$. (C) Optiprep density gradient analysis of detergent-insoluble fractions prepared from equal quantities (assessed by total protein) of WT and KO BBs; Coomassie Blue-stained gels loaded for equal volume are shown. Fraction 1 represents the top of the gradient (lowest density). Immunoblots for Myola (D), Myo1c (E), and SI (F) reveal that in the absence of Myo1a, total gradient levels of SI are slightly reduced and Myo1c is recruited into the lowest density, raft-rich fraction (1).

This apparent discrepancy may be related to the mode of Myola loss in each case. In the DN model system, all docking sites for Myola on the MV membrane are blocked, eliminating the potential for functional rescue by other myosin-I isoforms. In $\mathrm{KO}$ enterocytes, these same docking sites would be unoccupied, enabling the recruitment of other myosins-I and subsequent rescue of function. The striking ectopic recruitment of Myo1c to KO BBs supports this interpretation.

The concept of functional redundancy among class I myosins was first examined in studies with Dictyostelium that showed that resting cortical tension is controlled by multiple myosin I isoforms (Dai et al., 1999). To our knowledge, the recruitment of Myo1c to the $\mathrm{BB}$ in Myola $\mathrm{KO}$ mice is the first clear demonstration of this phenomenon in vertebrates. Myo1c is a reasonable candidate for restoring functions normally fulfilled by Myo1a; it binds multiple CaM light chains and possesses a short, yet highly basic C-terminal tail domain (34\% identical / 49\% similar relative to Myo1a) that can bind directly to acidic phospholipids (Tang et al., 2002). But despite marked redistribution into $\mathrm{KO} \mathrm{BBs}$, the degree of rescue accomplished by Myo1c is only partial as indicated by the obvious ultrastructural and compositional defects described above. The decreased effectiveness of Myolc could be related to a lower affinity for MV docking sites or a lower molar expression level in the BB. The latter is more than likely because lateral bridges are absent in KO MV, and the total amount of immunogens detected by the pan myosin-I CX-1 mAb is greatly reduced in the KO BBs.

Is it possible that the perturbations observed in $\mathrm{KO}$ mice are only indirectly related to the loss of Myola but directly related to the loss of other components? In this regard, Myo6 should receive the most consideration given its potential role in maintaining apical membrane morphology in the hair cell. In Snell's waltzer (sv) mice, which lack functional Myo6, hair cell differentiation is perturbed by the extensive fusion of stereocilia, a defect that ultimately leads to hair cell degeneration and a loss of hearing and vestibular function (Self et al., 1999). These authors also reported that enterocyte MV were shorter in $s v$ animals. To explain these defects, it was proposed that Myo6 plays a role in the tethering of plasma membrane at the base of protruding actin bundles (e.g., stereocilia or MV). Our laboratory also has initiated an extensive phenotypic characterization of $s v$ mice, with a focus on the intestinal BB. Although our preliminary findings do suggest the presence of apical membrane defects, the frequency and severity do not approach that observed in Myola KO mice. Only after careful comparison of Myola $\mathrm{KO}$ and sv phenotypes will we be able to parse out the relative contributions of these two myosins to enterocyte function. 
Myo1a bridges were first observed in electron micrographs of enterocytes and isolated BBs $\sim 30$ years ago (Mukherjee and Staehelin, 1971; Mooseker and Tilney, 1975; Matsudaira and Burgess, 1979). These studies are the first to examine the consequences of eliminating Myola expression in an in vivo context. The diverse array of phenotypes observed in $\mathrm{KO}$ mice indicates that Myola is a multifunctional motor, required for maintaining the normal composition and exquisite organization of the enterocyte BB. Future studies must now focus on uncovering the molecular mechanisms of the phenotypes described here and the contributions of Myola to specific aspects of enterocyte structure and function.

\section{ACKNOWLEDGMENTS}

We thank members of the Mooseker laboratory for helpful suggestions, Peter Gillespie for the gift of anti-Myolc antibodies, and Andreas Quaroni for the gift of anti-SI antibodies. This work was supported by National Institutes of Health grants DK-25387 and DK-55389 (to M.S.M.), National Research Service Award DK-10113 (to M.J.T.), a Crohn's and Colitis Foundation of America Career Development Award (to M.J.T.), Training Program in Investigative Gastroenterology Postdoctoral Fellowship DK-07017 (to A.T.M.), and funds from the National Cancer Institute, U.S. Department of Health and Human Services (to N.A.J. and N.G.C.).

\section{REFERENCES}

Altman, D., Sweeney, H. L., and Spudich, J. A. (2004). The mechanism of myosin VI translocation and its load-induced anchoring. Cell 116, 737-749.

Berg, J. S., Powell, B. C., and Cheney, R. E. (2001). A millennial myosin census. Mol. Biol. Cell 12, 780-794.

Braccia, A., Villani, M., Immerdal, L., Niels-Christiansen, L. L., Nystrom, B. T., Hansen, G. H., and Danielsen, E. M. (2003). Microvillar membrane microdomains exist at physiological temperature. Role of galectin-4 as lipid raft stabilizer revealed by "superrafts." J. Biol. Chem. 278, 15679-15684.

Brown, D. A., and London, E. (1998). Functions of lipid rafts in biological membranes. Annu. Rev. Cell Dev. Biol. 14, 111-136.

Burgess, D. R., and Prum, B. E. (1982). Reevaluation of brush border motility: calcium induces core filament solution and microvillar vesiculation. J. Cell Biol. 94, 97-107.

Caradonna, L., Amati, L., Magrone, T., Pellegrino, N. M., Jirillo, E., and Caccavo, D. (2000). Enteric bacteria, lipopolysaccharides and related cytokines in inflammatory bowel disease: biological and clinical significance. J. Endotoxin Res. 6, 205-214.

Carboni, J. M., Conzelman, K. A., Adams, R. A., Kaiser, D. A., Pollard, T. D. and Mooseker, M. S. (1988). Structural and immunological characterization of the myosin-like 110-kD subunit of the intestinal microvillar 110K-calmodulin complex: evidence for discrete myosin head and calmodulin-binding domains. J. Cell Biol. 107, 1749-1757.

Dai, J., and Sheetz, M. P. (1999). Membrane tether formation from blebbing cells. Biophys. J. 77, 3363-3370.

Dai, J., Ting-Beall, H. P., Hochmuth, R. M., Sheetz, M. P., and Titus, M. A. (1999). Myosin I contributes to the generation of resting cortical tension. Biophys. J. 77, 1168-1176.

Danielsen, E. M., and Hansen, G. H. (2003). Lipid rafts in epithelial brush borders: atypical membrane microdomains with specialized functions. Biochim. Biophys. Acta 1617, 1-9.

Ding, M., Eliasson, C., Betsholtz, C., Hamberger, A., and Pekny, M. (1998). Altered taurine release following hypotonic stress in astrocytes from mice deficient for GFAP and vimentin. Mol. Brain Res. 62, 77-81.

Donaudy, F., Ferrara, A., Esposito, L., Hertzano, R., Ben-David, O., Bell, R. E., Melchionda, S., Zelante, L., Avraham, K. B., and Gasparini, P. (2003). Multiple mutations of MYO1A, a cochlear-expressed gene, in sensorineural hearing loss. Am. J. Hum. Genet. 72, 1571-1577.

Durrbach, A., Collins, K., Matsudaira, P., Louvard, D., and Coudrier, E. (1996). Brush border myosin-I truncated in the motor domain impairs the distribution and the function of endocytic compartments in an hepatoma cell line. Proc. Natl. Acad. Sci. USA 93, 7053-7058.

Durrbach, A., Raposo, G., Tenza, D., Louvard, D., and Coudrier, E. (2000). Truncated brush border myosin I affects membrane traffic in polarized epithelial cells. Traffic 1, 411-424.
Espindola, F. S., Cheney, R. E., King, S. M., Suter, D. M., and Mooseker, M. S (1996). Myosin-V and dynein share a similar light chain (Abstract). Mol. Biol. Cell 7, 372 .

Estivariz, C. F., Jonal, C. R., Gu, L. H., Diaz, E. E., Wallace, T. M., Pascal, R. R., Farrell, C. L., and Ziegler, T. R. (1998). Gut-trophic effects of keratinocyte growth factor in rat small intestine and colon during enteral refeeding. JPEN J. Parenter. Enteral Nutr. 22, 259-267.

Fath, K. R., Trimbur, G. M., and Burgess, D. R. (1994). Molecular motors are differentially distributed on Golgi membranes from polarized epithelial cells. J. Cell Biol. 126, 661-675.

Ferrary, E., et al. (1999). In vivo, villin is required for $\left.\mathrm{Ca}^{2+}\right)$-dependent F-actin disruption in intestinal brush borders. J. Cell Biol. 146, 819-830.

Gilbert, S., Loranger, A., Daigle, N., and Marceau, N. (2001). Simple epithelium keratins 8 and 18 provide resistance to Fas-mediated apoptosis. The protection occurs through a receptor-targeting modulation. J. Cell Biol. 154, $763-773$

Hasson, T. (2003). Myosin VI: two distinct roles in endocytosis. J. Cell Sci. 116 3453-3461.

Hasson, T., and Mooseker, M. S. (1994). Porcine myosin-VI: characterization of a new mammalian unconventional myosin. J. Cell Biol. 127, 425-440.

Heintzelman, M. B., and Mooseker, M. S. (1990a). Assembly of the brush border cytoskeleton: changes in the distribution of microvillar core proteins during enterocyte differentiation in adult chicken intestine. Cell Motil. Cytoskeleton 15, 12-22.

Heintzelman, M. B., and Mooseker, M. S. (1990b). Structural and compositional analysis of early stages in microvillus assembly in the enterocyte of the chick embryo. Differentiation 43, 175-182.

Hunziker, W., Spiess, M., Semenza, G., and Lodish, H. F. (1986). The sucraseisomaltase complex: primary structure, membrane-orientation, and evolution of a stalked, intrinsic brush border protein. Cell 46, 227-234.

Jontes, J. D., Milligan, R. A., Pollard, T. D., and Ostap, E. M. (1997). Kinetic characterization of brush border myosin-I ATPase. Proc. Natl. Acad. Sci. USA 94, 14332-14337.

Kuisma, J., Nuutinen, H., Luukkonen, P., Jarvinen, H., Kahri, A., and Farkkila, M. (2001). Long term metabolic consequences of ileal pouch-anal anastomosis for ulcerative colitis. Am. J. Gastroenterol. 96, 3110-3116.

Mammen, J. M., and Matthews, J. B. (2003). Mucosal repair in the gastrointestinal tract. Crit. Care Med. 31, S532-537.

Matsudaira, P. T., and Burgess, D. R. (1979). Identification and organization of the components in the isolated microvillus cytoskeleton. J. Cell Biol. 83, 667-673.

Mooseker, M. S. (1985). Organization, chemistry, and assembly of the cytoskeletal apparatus of the intestinal brush border. Annu. Rev. Cell Biol. 1, 209-241, 209-241.

Mooseker, M. S., and Cheney, R. E. (1995). Unconventional myosins. Ann. Rev. Cell Dev. Biol. 11, 633-675, 633-675.

Mooseker, M. S., Keller, T. C., and Hirokawa, N. (1983). Regulation of cytoskeletal structure and contractility in the brush border. Ciba Found. Symp. 95, 195-215, 195-215.

Mooseker, M. S., and Tilney, L. G. (1975). Organization of an actin filamentmembrane complex. Filament polarity and membrane attachment in the microvilli of intestinal epithelial cells. J. Cell Biol. 67, 725-743.

Mukherjee, T. M., and Staehelin, L. A. (1971). The fine-structural organization of the brush border of intestinal epithelial cells. J. Cell Sci. 8, 573-599.

Okayasu, I., Hatakeyama, S., Yamada, M., Ohkusa, T., Inagaki, Y., and Nakaya, R. (1990). A novel method in the induction of reliable experimental acute and chronic ulcerative colitis in mice. Gastroenterology 98, 694-702.

Pearl, M., Fishkind, D., Mooseker, M., Keene, D., and Keller, T. (1984). Studies on the spectrin-like protein from the intestinal brush border, TW 260/240, and characterization of its interaction with the cytoskeleton and actin. J. Cell Biol. $98,66-78$.

Sasahara, T., Maruyama, H., Aoki, M., Kikuno, R., Sekiguchi, T., Takahashi, A., Satoh, Y., Kitasato, H., Takayama, Y., and Inoue, M. (2003). Apoptosis of intestinal crypt epithelium after Cryptosporidium parvum infection. J. Infect. Chemother. 9, 278-281.

Self, T., Sobe, T., Copeland, N. G., Jenkins, N. A., Avraham, K. B., and Steel, K. P. (1999). Role of myosin VI in the differentiation of cochlear hair cells. Dev. Biol. 214, 331-341.

Sharp, D. J., Rogers, G. C., and Scholey, J. M. (2000). Microtubule motors in mitosis. Nature $407,41-47$. 
Sheetz, M. P. (2001). Cell control by membrane-cytoskeleton adhesion. Nat. Rev. Mol. Cell. Biol. 2, 392-396.

Skowron, J. F., Bement, W. M., and Mooseker, M. S. (1998). Human brush border myosin-I and myosin-Ic expression in human intestine and Caco-2BBe cells. Cell Motil. Cytoskeleton 41, 308-324.

Taminiau, J. A., Gall, D. G., and Hamilton, J. R. (1980). Response of the rat small-intestine epithelium to methotrexate. Gut 21, 486-492.

Tang, N., Lin, T., and Ostap, E. M. (2002). Dynamics of myo1c (myosin-i $\beta$ ) lipid binding and dissociation. J. Biol. Chem. 277, 42763-42768.

Tyska, M. J., and Mooseker, M. S. (2002a). MYO1A (brush border myosin I) dynamics in the brush border of LLC-PK1-CL4 cells. Biophys. J. 82, 18691883.
Tyska, M. J., and Mooseker, M. S. (2002b). Overexpression of myosin-IA tail domain abolishes microvillar length regulation and disrupts the localization of myosin-VI. A.S.C.B. Annual Meeting (Abstract 1006).

Tyska, M. J., and Mooseker, M. S. (2004). A role for myosin-1A in the localization of a brush border disaccharidase. J. Cell Biol. 165, 395-405.

Veigel, C., Coluccio, L. M., Jontes, J. D., Sparrow, J. C., Milligan, R. A., and Molloy, J. E. (1999). The motor protein myosin-I produces its working stroke in two steps. Nature $398,530-533$

Wells, A. L., Lin, A. W., Chen, L. Q., Safer, D., Cain, S. M., Hasson, T., Carragher, B. O., Milligan, R. A., and Sweeney, H. L. (1999). Myosin VI is an actin-based motor that moves backwards. Nature 401, 505-508.

Wilson, S. M., et al. (2000). The status of voltage-dependent calcium channels in alpha $1 \mathrm{E}$ knock-out mice. J. Neurosci. 20, 8566-8571. 